\title{
Statistically Deformable 2D/3D Registration for Estimating Post-operative Cup Orientation from a Single Standard AP X-ray Radiograph
}

\author{
GuOyan Zheng \\ Institute for Surgical Technology and Biomechanics, University of Bern, Stauffacherstrasse 78, CH-3014 Bern, Switzerland
}

(Received 13 September 2009; accepted 30 April 2010; published online 16 June 2010)

Associate Editor Larry V. McIntire oversaw the review of this article.

\begin{abstract}
The widely used procedure of estimating postoperative cup orientation based on a single standard AP $\mathrm{X}$-ray radiograph is known inaccurate, largely due to the wide variability in individual pelvic orientation relative to $\mathrm{X}$-ray plate. CT-based $2 \mathrm{D} / 3 \mathrm{D}$ rigid image registration methods have been developed to measure post-operative cup orientation. Although encouraging results have been reported, their extensive usage in clinical routine is still limited. This may be explained by their requirement of having a CT study of the patient at some point during treatment, which is not available for vast majority of Total Hip Arthroplasty procedures performed nowadays. To address this limitation, this article proposes a statistically deformable $2 \mathrm{D} / 3 \mathrm{D}$ registration approach for estimating post-operative cup orientation. No CT study of the patient is required any more. Compared to ground truths established from post-operative CT images, the cup orientations measured by the present technique in a cadaver experiment showed differences of $1.7 \pm 1.4^{\circ}$ for anteversion and difference of $1.5 \pm 1.5^{\circ}$ for inclination. When the present technique was evaluated on patients' datasets, differences of $2.2 \pm 1.3^{\circ}$ and differences of $2.0 \pm 0.8^{\circ}$ were found for the anteversion and the inclination, respectively. The experimental results, though still preliminary, demonstrated the efficacy of the present approach.
\end{abstract}

Keywords-Statistical shape model, Point distribution model, Statistically deformable $2 \mathrm{D} / 3 \mathrm{D}$ registration, X-ray radiograph, Post-operative cup orientation, Pelvis.

\section{ABBREVIATIONS}

\section{THA Total Hip Arthroplasty}

AP Antero-Posterior

Address correspondence to Guoyan Zheng, Institute for Surgical Technology and Biomechanics, University of Bern, Stauffacherstrasse 78, CH-3014 Bern, Switzerland. Electronic mail: guoyan. zheng@ieee.org, guoyan.zheng@istb.unibe.ch

$\begin{array}{ll}\text { 3D } & \text { Three-dimensional } \\ \text { 2D } & \text { Two-dimensional } \\ \text { CT } & \text { Computed Tomography } \\ \text { APP } & \text { Anterior Pelvic Plane } \\ \text { ASIS } & \text { Anterior Superior Iliac Spine } \\ \text { SSM } & \text { Statistical Shape Model } \\ \text { DRR } & \text { Digitally Reconstructed Radiograph } \\ \text { PDM } & \text { Point Distribution Model } \\ \text { DICOM } & \text { Digital Imaging and COmmunication } \\ & \text { in Medicine } \\ \text { PCA } & \text { Principal Component Analysis } \\ \text { LM } & \text { Lateral-Medial }\end{array}$

\section{INTRODUCTION}

Proper component positioning is essential for Total Hip Arthroplasty (THA) in regards to both the shortterm complications and long-term survival results. ${ }^{1,3,30,34,41,46,58}$ Previous studies demonstrate that the higher rates of pelvis osteolysis, the severity of the polyethylene wear, and the component migration have all been well associated with the mal-positioning of the acetabular component, ${ }^{1,3,30,34,41,46,58}$ and surgical experience indicates that the mal-orientation of the acetabular component in terms of anteversion and inclination is the major cause of dislocation. ${ }^{1,30,34,41,46,58}$ It is thus very important to develop an accurate method to measure the post-operative cup orientation for a reasonable follow-up after THA.

Two-dimensional (2D) anteroposterior (AP) pelvic radiograph is the standard imaging means for measuring the post-operative cup orientation. ${ }^{2,12,22,33,44,51,57}$ Although it has an inferior accuracy in comparison to three-dimensional (3D) 
techniques based on Computed Tomography (CT) ${ }^{2,22,33}$ it is used routinely because of its simplicity, availability, and minimal expense associated with its acquisition. While plain pelvic radiographs are easily obtained, their accurate interpretations are subjected to substantial errors if the individual pelvis orientation with respect to X-ray plate is not taken into consideration. ${ }^{2,22,33}$ For example, the increased pelvic tilt will result in significant decreases in apparent prosthetic cup anteversion and vice versa. ${ }^{47}$ Improved methods of measuring component positions post-operatively are essential for the accuracy of studies correlation cup position to osteolysis, wear, and instability, for evaluation of surgical treatment techniques and treatment methods, and for our ability to determine optimal range of the component positioning.

When a CT study of the patient is available at some point during treatment, CT-based $2 \mathrm{D} / 3 \mathrm{D}$ rigid image registration methods, $8,20,28,42,49,64,65$ including the method proposed by my own group, ${ }^{49,64,65}$ have been developed to measure the post-operative cup orientation with respect to an anatomical reference extracted from the CT images, which is a plane called the Anterior Pelvic Plane (APP) defined by the Anterior Superior Iliac Spines (ASIS) and the pubic tubercles. ${ }^{19,26,50}$ In such methods, a rigid transformation between the $\mathrm{CT}$ data coordinate system and the X-ray image coordinate system is estimated first by performing an intensity-based rigid $2 \mathrm{D} / 3 \mathrm{D}$ registration, which then allows for computing the orientation of the acetabular cup with respect to the APP extracted from the CT images.

While accurate, ${ }^{8,20,42,49,64,65}$ the extensive usage of the CT-based $2 \mathrm{D} / 3 \mathrm{D}$ image registration methods in clinical routine is still limited. This may be explained by their requirement of having a CT study of the patient at some point during treatment, which is not available for vast majority of THA procedures performed nowadays ${ }^{42}$ or for earlier cup design. In this article, a statistical shape mode-based $2 \mathrm{D} / 3 \mathrm{D}$ reconstruction method is proposed to address such a limitation. The basic idea behind the present method is to formulate the problem of estimating post-operative cup orientation as a process coupling a radiographic measurement with a $2 \mathrm{D} / 3 \mathrm{D}$ reconstruction, where a patient-specific 3D model will be reconstructed from the X-ray image, thus eliminating the requirement of having a CT study of the patient.

It is well-known that constructing a patient-specific 3D model from 2D X-ray image(s) is a challenging task. A priori information is often required to handle this otherwise ill-posed problem. Previously, krigingbased methods, ${ }^{18,21,27,29,36-38,40,43}$ as well as statistical shape model (SSM) based methods, ${ }^{5,6,16,24,25,32,45}$ have been proposed.
Kriging-based methods start with establishing image-to-model correspondences by finding the corresponding anatomical landmarks, ${ }^{18,36,37,43}$ or contour points. ${ }^{21,27,29,38,40}$ The reconstruction is done by first estimating the scale and the rigid transformation of a generic object with respect to features extracted from the input images and then applying a kriging algorithm to deform the generic object to obtain the final surface model.

Instead of using one generic object as the prior information, the methods in the second category use statistical shape models obtained from statistical shape analysis. Statistical shape analysis is an important tool for understanding anatomical structures from medical images. ${ }^{14,23,48}$ Statistical shape models give efficient parameterizations of the shape variations found in a collection of sample models of a given population. Model-based approaches are popular due to their ability to robustly represent objects. ${ }^{10,55}$ In the last few years, constructing a patient-specific shape model from a limited number of calibrated X-ray images and a SSM has drawn more and more attentions. $5,6,16,24,25,32,45,60,62,63$ Except the method presented by Sadowsky et al., ${ }^{45}$ and the method presented by Mahfouz et al., ${ }^{32}$ which depend on a deformable 2D/3D registration between the digitally reconstructed radiographs (DRRs) of a SSM and the X-ray images, all other methods have their reliance on feature-based statistical instantiation from a Point Distribution Model (PDM) in common. 5,6,16,24,25,60,62,63

Common to all these previous works on reconstructing a patient-specific 3D model from X-ray images are: (a) at least two images are used ${ }^{5,6,16,18,21,24,25,27,29,32,36-38,43,45,60,62,63}$; and/or (b) all images are calibrated. ${ }^{5,6,16,18,21,24,25,27,29,32,36-38,40,43,45,60,62,63}$ However, in the target application reported in this article, no radiograph-specific calibration is available. The only information that one can assume to know about the radiograph is the image scale $(\mathrm{mm} / \mathrm{pixel})$ and the distance from the focal point to the imaging plane or to the film (it is called the focal-point-to-film distance). As long as the radiograph is acquired in a standardized way, which is performed in a clinical routine, ${ }^{12}$ both parameters can be retrieved directly from the DICOM header if the X-ray image is stored in DICOM format (this is the way how those two parameters were obtained for all experiments reported in this study), or can be estimated by performing a one-time calibration using a calibration object of known size. ${ }^{52}$ When only a single image is used, it is well-known that the depth information and the scaling factor are correlated with each other. Thus, precise estimation of both of them from a single image is difficult. However, considering the context of our application, one can hypothesize 
that even a scaled estimation of the pelvis model should provide enough information to determine the post-operative cup orientation. Thus, in this article, a statistically deformable $2 \mathrm{D} / 3 \mathrm{D}$ registration approach is proposed to instantiate a patient-specific surface model of the pelvis from the single standard AP X-ray radiograph for estimating the post-operative cup orientation. $^{59}$

\section{METHODS}

\section{Problem Formulation}

Unlike in previous work, ${ }^{8,20,28,42,64,65}$ the problem of estimating the post-operative cup orientation in this article is formulated as a process coupling a radiographic measurement with a statistically deformable 2D/3D reconstruction, which can be expressed as (see Fig. 1 for details),

$$
\vec{n}_{\mathrm{APP}}=T_{\mathrm{R}}^{\mathrm{APP}-\text { Reconstructed Model }} \cdot \vec{n}_{\mathrm{R}}
$$

where $\vec{n}_{\mathrm{R}}$ means the orientation of the cup measured in the X-ray radiograph coordinate system; $T_{\mathrm{R}}^{\mathrm{APP}-\text { Reconstructed Model }}$ is the transformation between the local coordinate system of the X-ray radiograph and a cup orientation measurement coordinate system defined on the APP of a surface model that is reconstructed from the radiograph (see Fig. 1 for details). In order to calculate the post-operative cup orientation $\vec{n}_{\mathrm{APP}}$, Eq. (1) indicates that one can first compute the cup orientation with respect to the $\mathrm{X}$-ray radiograph coordinate system and then transform it to the coordinate system defined on the APP of a surface model that is derived from this X-ray radiograph. When this formulation is compared to other existing work, ${ }^{8,20,28,42,64,65}$ among the advantages such as the independence of a specific computer-aided design (CAD) model of the prosthesis and the elimination of a radiograph-specific calibration, the most significant advantage is the elimination of the necessity of possessing a CT study of the patient for an accurate measurement of the post-operative cup orientation.

When a surface model has already been reconstructed from the X-ray radiograph, there already exist methods for computing $\vec{n}_{\mathrm{R}}$ and $T_{\mathrm{R}}^{\mathrm{APP}-\text { Reconstructed Model }}$. In this study, the method introduced in Widmer ${ }^{57}$ was used to find $\vec{n}_{\mathrm{R}}$ and the method published in Jaramaz et $a l .{ }^{19}$ was used to find $T_{\mathrm{R}}^{\mathrm{APP}-\text { Reconstructed Model }}$. The challenge then lies in the derivation of a patient-specific surface model from the AP X-ray radiograph, which is solved by a statistically deformable $2 \mathrm{D} / 3 \mathrm{D}$ registration (see section "Statistically Deformable 2D/3D Registration" for details).

\section{Construction of the Statistical Shape Model of the Pelvis}

The PDM was chosen as the representation of the statistical shape model of the pelvis. The pelvic PDM used in this study was constructed from a training database consisting of 14 segmented binary volumes (12 of them were segmented from CT scans of

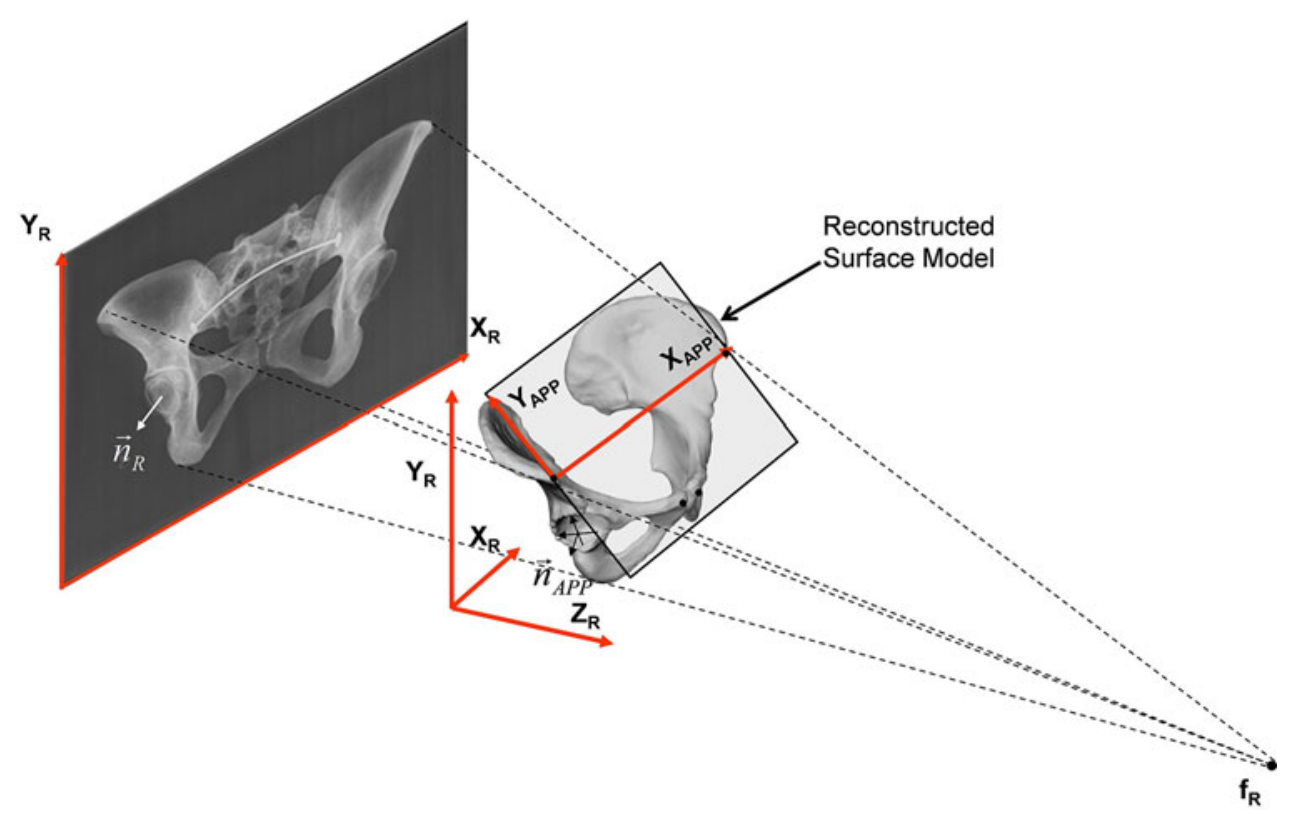

FIGURE 1. Schematic representation of using a surface model that is reconstructed from a single standard AP X-ray radiograph for estimating the post-operative cup orientation. 
cadaveric pelvises and the rest 2 were segmented from CT scans of patient's pelvises) where the sacrum was removed from each dataset. After one of the binary volumes was chosen as the reference, Demon's algorithm, ${ }^{53}$ as implemented in MedINRIA,${ }^{54}$ was used to estimate the dense deformation fields between the reference binary volume and the other 13 binary volumes. Each estimated deformation field was then used to displace the positions of the vertices on the reference surface model, which was constructed from the reference binary volume, to the associated target volume, resulting in 14 surface models with established correspondences.

Following the alignment, the PDM was constructed as follows. Let $\mathbf{x}_{i}, i=0,1, \ldots, m-1$ be $m$ (here $m=14)$ members in the aligned training population. Each member is described by a vector $\mathbf{x}_{i}$ containing $N$ vertices:

$$
\mathbf{x}_{i}=\left\{x_{0}, y_{0}, z_{0}, x_{1}, y_{1}, z_{1}, \ldots, x_{N-1}, y_{N-1}, z_{N-1}\right\}
$$

The PDM is constructed by applying principal component analysis (PCA) on these aligned vectors. ${ }^{11}$

$$
\begin{aligned}
\mathbf{D}= & \frac{1}{(m-1)} \cdot \sum_{i=0}^{m-1}\left(\mathbf{x}_{i}-\overline{\mathbf{x}}\right) \cdot\left(\mathbf{x}_{i}-\overline{\mathbf{x}}\right)^{T} \\
\mathbf{P}= & \left(\mathbf{p}_{0}, \mathbf{p}_{1}, \ldots, \mathbf{p}_{m-2}\right) ; \quad \mathbf{D} \cdot \mathbf{p}_{i}=\sigma_{i}^{2} \cdot \mathbf{p}_{i} \\
& \sigma_{0} \geq \sigma_{1} \geq \cdots \geq \sigma_{m-2}>0
\end{aligned}
$$

where $\overline{\mathbf{x}}$ and $\mathbf{D}$ represents the mean vector and the covariance matrix, respectively; $\left\{\sigma_{i}^{2}\right\}$ are non-zero eigenvalues of the covariance matrix $\mathbf{D}$, and $\left\{\mathbf{p}_{i}\right\}$ are the corresponding eigenvectors. The descendingly sorted eigenvalues $\sigma_{i}^{2}$ and the corresponding eigenvector $\mathbf{p}_{i}$ of the covariance matrix are the principal directions spanning a shape space with $\overline{\mathbf{x}}$ representing its origin. Figure 2 shows the variability captured by the first two modes of variations of the statistical model.

\section{Statistically Deformable 2D/3D Registration}

The single image-based surface model reconstruction technique proposed in this article is based on a hybrid $2 \mathrm{D} / 3 \mathrm{D}$ deformable registration process coupling a landmark-based scaled rigid registration with an adapted SSM-based 2D/3D reconstruction algorithm. ${ }^{60,62,63}$ Different from the situation in previous work, ${ }^{5,6,16,18,21,24,25,27,29,32,36-38,40,43,45,60,62,63}$ where two or more calibrated X-ray images were required as the input for a successful reconstruction, here only a single un-calibrated X-ray radiograph is available. In such a situation, how to establish the projection geometry of the input radiograph from the known information (the focal-point-to-film distance and the image scale) and how to initialize the statistical model with respect to the X-ray image are two main challenges. In the following, solutions to these two challenges are first presented before I present the statistical shape model-based $2 \mathrm{D} / 3 \mathrm{D}$ reconstruction method.

\section{Establishment of Projection Geometry}

The local coordinate frame and the cone-beam projection model of the AP X-ray radiograph are established as follows (see Fig. 3a for details). The image center is taken as the coordinate origin. The $X$-axis and the $Y$-axis of the image are taken as the $X$-axis and the $Y$-axis of the local coordinate frame, respectively. The central projection line is perpendicular to the image plane and its opposite direction is regarded as the $Z$-axis of the local coordinate frame.

\section{Landmark-based Scaled Rigid Registration for Initialization}

Initialization here means to estimate the initial scale and the initial rigid transformation between the mean model of the PDM and the input radiograph. For this purpose, an iterative landmark-to-ray scaled rigid
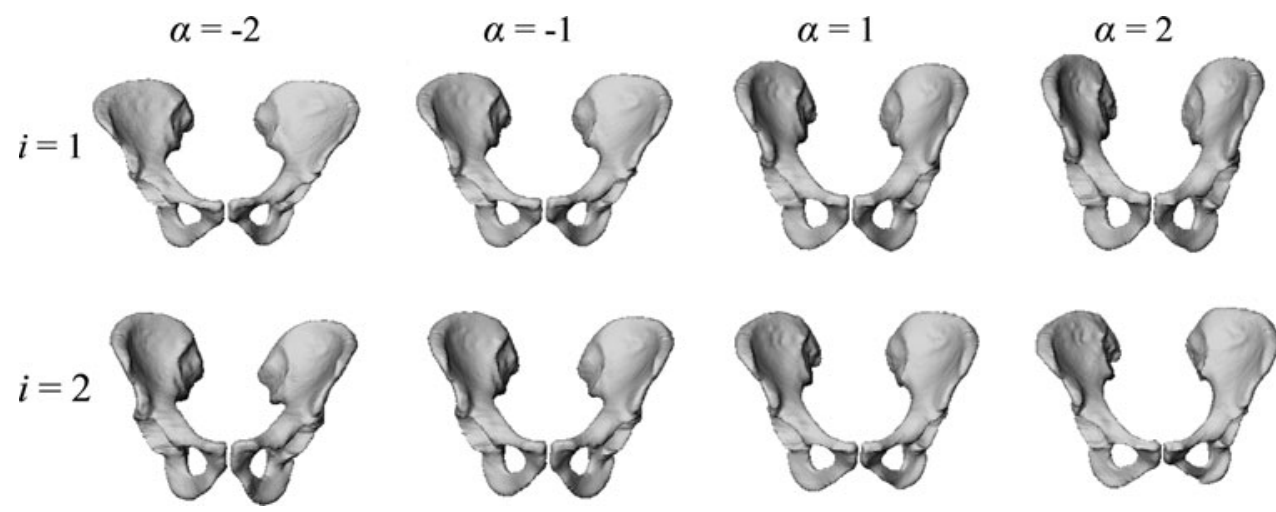

FIGURE 2. The first two eigen-modes of the variations of the PDM of the pelvis. The shape instances were generated by evaluating $\overline{\mathrm{x}}+\alpha \cdot \sigma_{i} \cdot \mathbf{p}_{i}$ with $\alpha \in\{-2,-1,1,2\}$. 

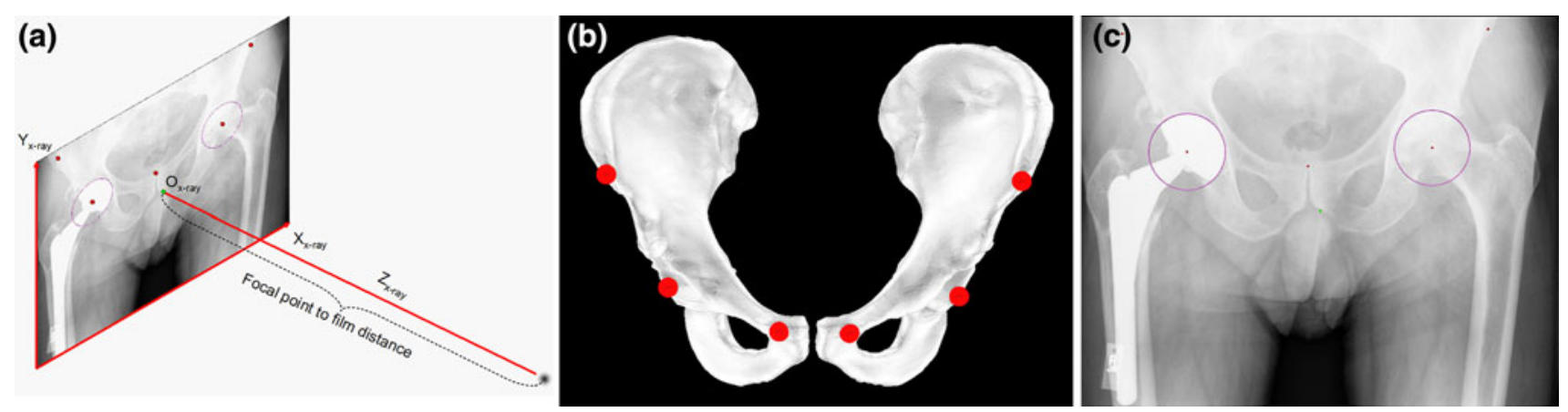

FIGURE 3. Schematic views of establishment of the projection geometry for a given AP X-ray radiograph and of the anatomical landmarks used in the present approach. (a) The radiograph coordinate system and the cone-beam projection model; (b) landmarks extracted from the mean model of the pelvic PDM; and (c) landmarks interactively picked from a radiograph.

registration is adopted. The five anatomical landmarks used in the scaled rigid registration are left and right ASIS, left and right acetabular centers, and the middle point of the left and right pubic tubercles. Their positions on the mean model of the PDM are obtained through point picking (for left and right ASIS, and for left and right pubic tubercles) or sphere fitting (for left and right acetabular centers), while their positions on the radiograph are defined through interactive picking (for the projections of left and right ASIS, and the center of the superior pubic symphysis) or circle fitting (for the projections of left and right acetabular centers) (see Figs. $3 b$ and $3 c$ for details).

Let me denote those landmarks defined on the mean model of the PDM, i.e., the left and the right acetabular centers, the middle point of the left and right pubic tubercles, and the middle point of the left and right ASIS, as $v_{\text {Mean }}^{1}, v_{\text {Mean }}^{2}, v_{\text {Mean }}^{3}$, and $v_{\text {Mean }}^{4}$, respectively, and their corresponding landmarks interactively picked from the radiograph as $v_{\mathrm{X}-\text { ray }}^{1}, v_{\mathrm{X}-\text { ray }}^{2}, v_{\mathrm{X}-\text {-ray }}^{3}$, and $v_{\mathrm{X} \text {-ray }}^{4}$ $\left(v_{\mathrm{X} \text {-ray }}^{4}\right.$ is the middle point of the projections of the left and the right ASIS), respectively. And for each X-ray landmark, one can calculate a projection ray emitting from the focal point to the landmark. Furthermore, one can calculate the length between $v_{\text {Mean }}^{1}$ and $v_{\text {Mean }}^{2}$ and denotes it as $l_{\text {Mean }}^{1,2}$. Using the known image scale, one can further calculate the length $l_{\mathrm{X}-\text { ray }}^{1,2}$ between $v_{\mathrm{X} \text {-ray }}^{1}$ and $v_{\mathrm{X} \text {-ray }}^{2}$. The iterative landmark-to-ray scaled rigid registration consists of two stages.

\section{Stage 1: Data Preparation}

In this step, one can assume that the line connecting the acetabular centers is parallel to the AP pelvic radiograph plane and is a certain distance away from the imaging plane (this distance is named as the objectto-film distance and in all the experiments reported in this article, a fixed object-to-film distance of $150 \mathrm{~mm}$ was used). Using this assumption and the correspondences between the landmarks defined in the CT volume and those from the radiograph, one can compute two points $\bar{v}_{\mathrm{X} \text {-ray }}^{1}$ and $\bar{v}_{\mathrm{X} \text {-ray }}^{2}$ on the projection rays of $v_{\mathrm{X} \text {-ray }}^{1}$ and $v_{\mathrm{X}-\text { ray }}^{2}$, respectively (see Fig. $4 \mathrm{a}$ for details), which satisfy:

$$
\begin{aligned}
& \bar{v}_{\mathrm{X}-\text { ray }}^{1} \bar{v}_{\mathrm{X} \text {-ray }}^{2} / / v_{\mathrm{X} \text {-ray }}^{1} v_{\mathrm{X} \text {-ray }}^{2} ; \\
& \text { and }\left|\bar{v}_{\mathrm{X} \text {-ray }}^{1}-\bar{v}_{\mathrm{X} \text {-ray }}^{2}\right|=l_{\mathrm{X} \text {-ray }}^{1,2} \times \frac{F-d}{F}
\end{aligned}
$$

where "//" is a parallel symbol and "|.|" means to compute the distance of two points; $F$ is the known focal-point-to-film distance; and $d$ is the assumed object-to-film distance.

The current scale $s$ between the mean model and the input image is then estimated as,

$$
s=\left|\bar{v}_{\mathrm{X}-\text { ray }}^{1}-\bar{v}_{\mathrm{X}-\text { ray }}^{2}\right| / l_{\text {Mean }}^{1,2}
$$

Using $s$, one can scale all landmark positions on the mean model and denotes them as $\left\{\bar{v}_{\text {Mean }}^{i} ; i=1,2,3,4\right\}$. Based on the scaled landmark positions, the distances from $\bar{v}_{\text {Mean }}^{3}$ and $\bar{v}_{\text {Mean }}^{3}$ to line $\bar{v}_{\text {Mean }}^{1} \bar{v}_{\text {Mean }}^{2}$ are calculated and are denoted as $\bar{l}_{\text {Mean }}^{3,1-2}$ and $\bar{l}_{\text {Mean }}^{4,1-2}$, respectively.

Next, one can find two points, point $\bar{v}_{\mathrm{X} \text {-ray }}^{3}$ on the projection ray of $v_{\mathrm{X} \text {-ray }}^{3}$ whose distance to the line $\bar{v}_{\mathrm{X}-\text { ray }}^{1} \bar{v}_{\mathrm{X} \text {-ray }}^{2}$ is equal to $\bar{l}_{\text {Mean }}^{3,1-2}$, and point $\bar{v}_{\mathrm{X} \text {-ray }}^{4}$ on the projection ray of $v_{\mathrm{X}-\text { ray }}^{4}$ whose distance to the line $\bar{v}_{\mathrm{X}-\text { ray }}^{1} \bar{v}_{\mathrm{X}-\text { ray }}^{2}$ is equal to $\bar{l}_{\text {Mean }}^{4,1-2}$. A paired-point matching performed on $\quad\left\{\bar{v}_{\text {Mean }}^{i} ; i=1,2,3,4\right\} \quad$ and $\left\{\bar{v}_{\mathrm{X}-\text { ray }}^{i} ; i=1,2,3,4\right\}$ is used to calculate an updated scale $s_{0}$ and a rigid transformation $\bar{T}_{\text {Mean }}^{\mathrm{X} \text {-ray }}$ (see Fig. $4 \mathrm{a}$ for details). ${ }^{56}$ From now on, one can assume that all information defined in the mean model coordinate frame has been transformed into the radiograph coordinate frame using $s_{0}$ and $\bar{T}_{\text {Mean }}^{\mathrm{X} \text {-ray }}$. Let me denote the transformed mean model landmarks as $\left\{\tilde{v}_{\text {Mean }}^{i}\right\}$.

\section{Stage 2: Iterative Landmark-to-Ray Registration} In this stage, the following steps are iteratively executed until convergence: 

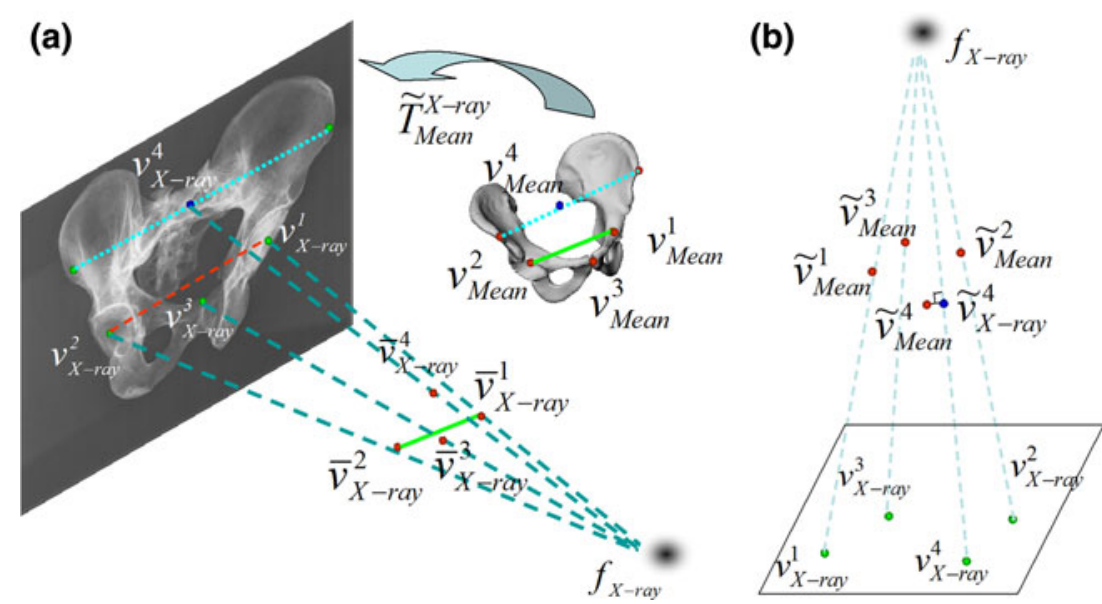

FIGURE 4. Schematic views of the iterative landmark-to-ray registration. (a) A schematic view of data preparation step; and (b) a schematic view of how to find 3D point pairs.
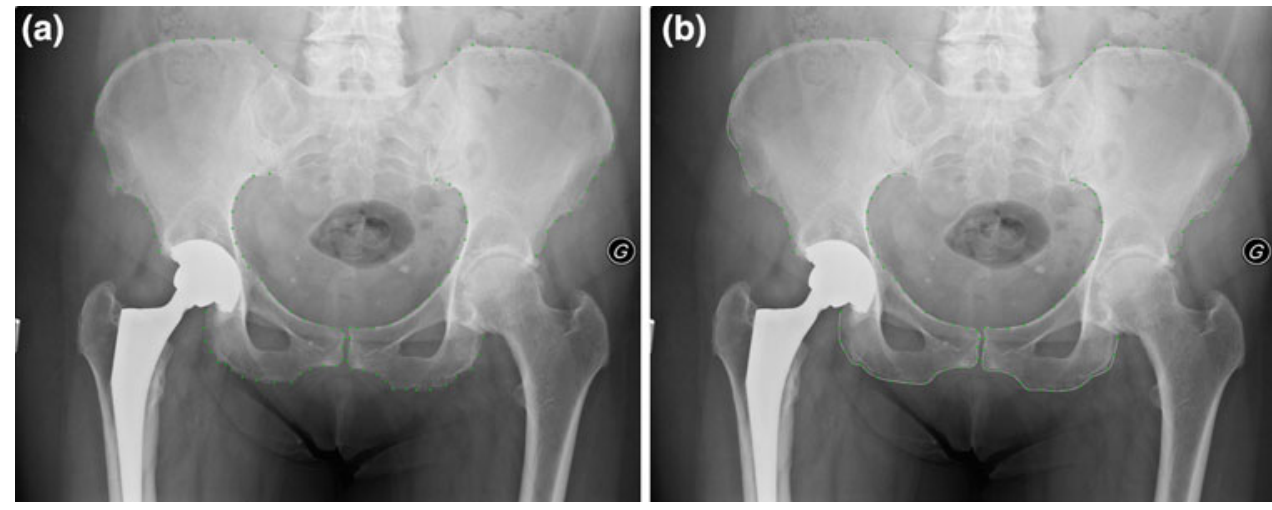

FIGURE 5. Interactive contour identification. (a) The interactively picked contour points (green spheres); and (b) the contours interpolated from the picked points with a cubic-spline (white lines).

1. For a point $\tilde{v}_{\text {Mean }}^{i}$, one can find a point on the corresponding projection ray of $v_{\mathrm{X}-\text { ray }}^{i}$ which has the shortest distance to the point $\tilde{v}_{\text {Mean }}^{i}$ and denote it as $\tilde{v}_{\mathrm{X}-\text { ray }}^{i}$ (see Fig. $4 \mathrm{~b}$ for details). A paired-point matching can then be performed on the extracted point pairs to compute an update $\Delta \tilde{s}$ for the scale and an update $\Delta \tilde{T}_{\text {Mean }}^{\mathrm{X}-\text { ray }}$ for the rigid transformation.

2. Update the mean model coordinate frame using $\Delta \tilde{s}$ and $\Delta \tilde{T}_{\text {Mean }}^{\mathrm{X}-\text { ray }}$.

\section{Statistical Shape Model-based 2D/3D Reconstruction}

The estimated scale and the rigid transformation between the mean model and the input image are treated as the starting values for the PDM-based 2D/ $3 \mathrm{D}$ reconstruction algorithm. This algorithm was first reported in Zheng et al. ${ }^{60,62,63}$ for reconstructing a patient-specific surface model of the proximal femur from multiple calibrated X-ray images. It is adapted here for reconstructing a scaled, patient-specific surface model of the pelvis from a single un-calibrated X-ray image. As a feature-based $2 \mathrm{D} / 3 \mathrm{D}$ reconstruction approach, this algorithm requires a pre-requisite image contour extraction. In this study, an interactive program was developed to allow the user to define up to eight contours by interactively picking points from the radiograph. The picked points for each contour are interpolated by a cubic-spline to have the same resolution as the image resolution. This procedure normally takes 2-4 min. Figure 5 shows an example of the picked contour points (Fig. 5a) and the interpolated continuous contours (Fig. 5b).

The extracted contours are then used together with the initial estimation of the scale and the rigid transformation as the input to the statistical shape modelbased 2D/3D reconstruction scheme, which combines statistical instantiation and regularized shape deformation with an iterative image-to-model correspondence establishing algorithm. The image-to-model 
correspondences are established using a non-rigid 2D point matching process, ${ }^{62}$ which iteratively uses a symmetric injective nearest-neighbor mapping operator and 2D thin-plate splines-based deformation to find a fraction of best matched $2 \mathrm{D}$ point pairs between features extracted from the X-ray images and the projections of the apparent contours extracted from the $3 \mathrm{D}$ model. The apparent contours of a statistically instantiated 3D model are extracted using the approach introduced by Hertzmann and Zorin. ${ }^{17}$ Previously, it was mathematically proved that the proposed non-rigid $2 \mathrm{D}$ point matching process could automatically eliminate the cross-matching event, ${ }^{62}$ which was defined as the interactions between the lines linking any matched point pair. Figure 6 a shows the mean mode of the pelvic PDM initialized with respect to the input image using the landmark-based scaled rigid registration, and the apparent contours extracted from the mean model. An example of building 2D/2D correspondences between the image contours and the projections of the apparent contours of the mean model is presented in Fig. 6b. The obtained 2D point pairs are then used to set up a set of $3 \mathrm{D}$ point pairs so that one can turn a $2 \mathrm{D} / 3 \mathrm{D}$ reconstruction problem to a $3 \mathrm{D} / 3 \mathrm{D}$ one. For details about how the proposed nonrigid $2 \mathrm{D}$ point matching process works and about the mathematic proof of how the proposed process eliminates the cross-matching event, I refer to the previous work. ${ }^{62}$ In the following, the details about how to convert the $2 \mathrm{D} / 3 \mathrm{D}$ reconstruction problem to a $3 \mathrm{D} / 3 \mathrm{D}$ one and how the latter problem is solved are given for completeness. Figure $6 \mathrm{c}$ shows an example of the 2D/ $3 \mathrm{D}$ reconstruction result.

A. Converting a $2 D / 3 D$ Problem to a $3 D / 3 D$ one Assume that a set of $2 \mathrm{D}$ matched point pairs $\left\{\left(A_{b}, I_{b}\right) ; b=0,1, \ldots, n-1\right\}$ have been found, where
$A_{b}$ is the projection of a point on the apparent contours of a $3 \mathrm{D}$ model that is instantiated from the PDM and $I_{b}$ is a point on the image contours that is matched to $A_{b}$. The corresponding 3D point pairs are then constructed as follows (see Fig. 7 for a schematic illustration). For a $2 \mathrm{D}$ point $I_{b}$, one can find a projection ray $r_{b}$ emitting from the focal point of the X-ray image through the point $I_{b}$. Additionally, for its matched point $A_{b}$, one knows the associated $3 \mathrm{D}$ point $\Omega_{b}$ on the apparent contours of the model whose projection onto the image is $A_{b}$. By computing a point $v_{b}$ on the ray $r_{b}$ that has the shortest distance to $\Omega_{b}$, a $3 \mathrm{D}$ point pair $\left(\Omega_{b}, v_{b}\right)$ can be obtained. Combining all these $3 \mathrm{D}$ point pairs, one can establish $2 \mathrm{D} / 3 \mathrm{D}$ correspondence between the input image and a $3 \mathrm{D}$ model instantiated from the PDM, and thus convert a 2D/3D reconstruction problem into a $3 \mathrm{D} / 3 \mathrm{D}$ one.

\section{B. 3D/3D Reconstruction}

As soon as a set of $3 \mathrm{D}$ point pairs are available, the problem of surface reconstruction is then solved optimally in three stages using the algorithm presented in Zheng et al. ${ }^{61,62}$ : scaled rigid registration, statistical instantiation, and regularized shape deformation.

Scaled rigid registration This is the only stage that is solved iteratively. In this stage, the scale and the rigid registration transformation between the mean model of the PDM and the input images are iteratively determined using an adapted iterative closest point (ICP) algorithm. ${ }^{7}$ The difference between this algorithm and the traditional ICP algorithm is that at each iteration one need to set up a set of new point pairs using the algorithm for building $2 \mathrm{D} / 3 \mathrm{D}$ correspondences. ${ }^{62}$

Statistical instantiation Based on the estimated scale and the pose information from the first stage, one can use the same correspondence establishing algorithm to
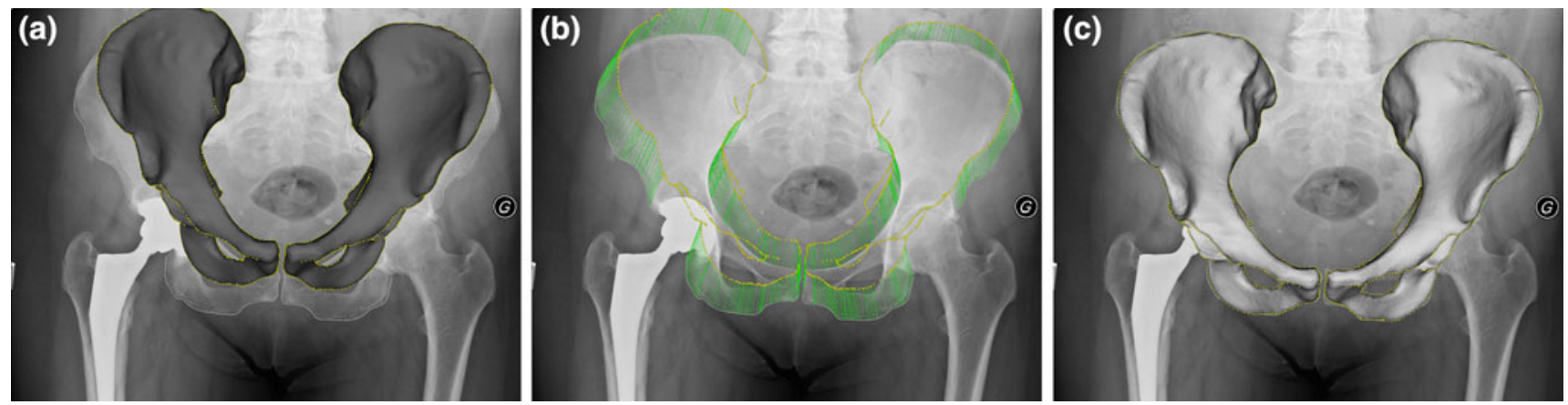

FIGURE 6. Screenshots of landmark-based initialization, of the establishment of 2D/2D correspondences, and of the 2D/3D reconstruction result. (a) The mean model of the pelvic PDM (dark gray surface model) initialized with respect to the input image using the landmark-based scaled rigid registration, and the apparent contours (yellow dots) extracted from the mean model; (b) the establishment of 2D/2D correspondences between the projections of the apparent contours (yellow) and the extracted image contours (white). The corresponding points are linked with green line segments for visualization purpose; and (c) an example of the $2 \mathrm{D} / 3 \mathrm{D}$ reconstruction result. 


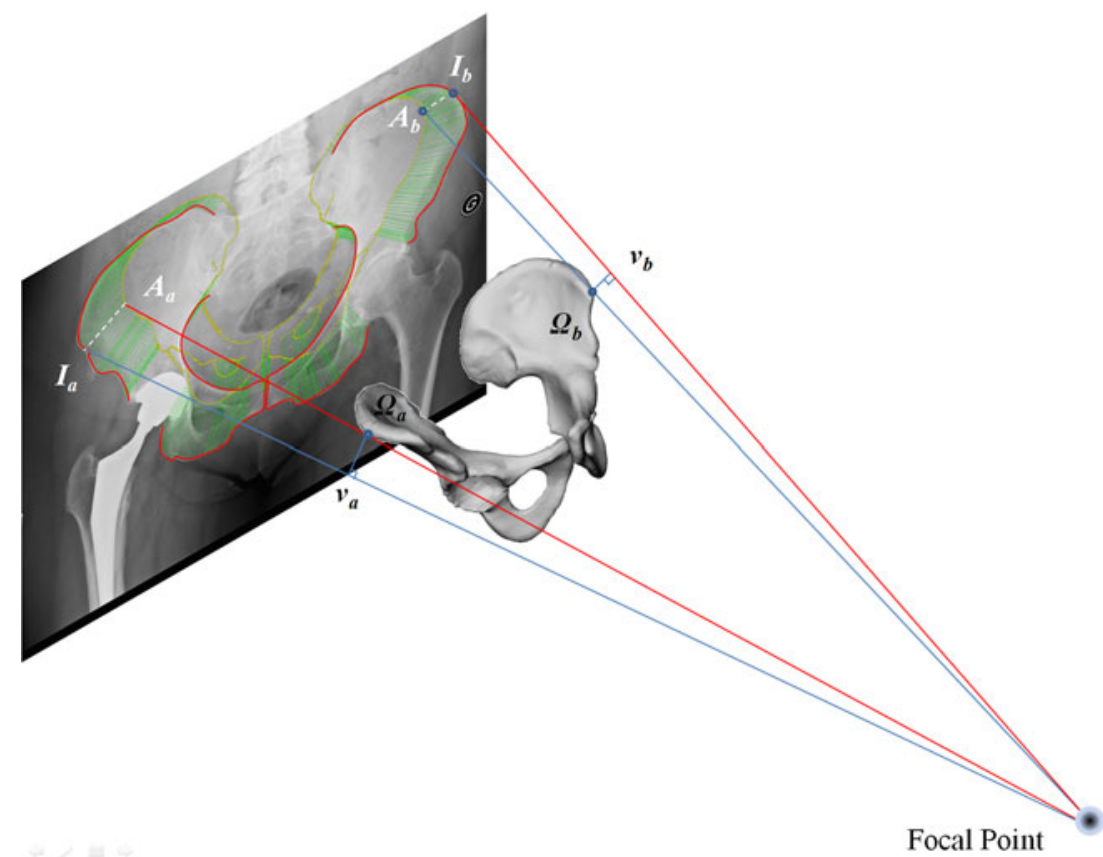

FIGURE 7. Schematic illustration of computing 3D point pairs between the model and the input image from the established 2D/2D correspondences.

obtain a set of $n 3 \mathrm{D}$ point pairs from the input image. Let me denote the points computed from the image data as $\mathbf{v}^{\prime}=\left\{v_{i}^{\prime}=\left(x_{i}^{\prime}, y_{i}^{\prime}, z_{i}^{\prime}\right) ; i=0,1, \ldots, n-1\right\}$ and call them the image points. Let me further denote those points on the mean model of the PDM as $\left.\overline{\mathbf{x}}^{\prime}=\left\{\overline{\mathbf{x}}_{j}\right)_{i} ; 0 \leq j \leq N-1 ; i=0,1, \ldots, n-1\right\}$ and call them the model points, where $N$ is the number of points on the mean model; $n$ is the number of the matched point pairs. $\left(\overline{\mathbf{x}}_{j}\right)_{i}$ means that the $j$ th model point $\overline{\mathbf{x}}_{j}$ on the mean model $\overline{\mathbf{x}}$ is the closest point to the $i$ th image point $v_{i}^{\prime}$. The statistical instantiation is formulated as the minimization of the following joint cost function $^{61,62}$ :

$$
\left\{\begin{array}{l}
E_{\alpha}\left(\overline{\mathbf{x}}^{\prime}, \mathbf{v}^{\prime}, \mathbf{x}\right)=(\rho+\log (3 n)) \cdot E\left(\overline{\mathbf{x}}^{\prime}, \mathbf{v}^{\prime}, \mathbf{x}\right)+E(\mathbf{x}) \\
\quad \mathbf{x}=\overline{\mathbf{x}}+\sum_{k=0}^{m-2} \alpha_{k} \cdot \sigma_{k} \cdot \mathbf{p}_{k} \\
E\left(\overline{\mathbf{x}}^{\prime}, \mathbf{v}^{\prime}, \mathbf{x}\right)=(1 / n) \cdot \sum_{i=0}^{n-1}\left\|v_{i}^{\prime}-\left(\left(\overline{\mathbf{x}}_{j}\right)_{i}+\sum_{k=0}^{m-2} \alpha_{k} \cdot \sigma_{k} \cdot \mathbf{p}_{k}(j)\right)\right\|^{2} \\
E(\mathbf{x})=(1 / 2) \cdot \sum_{k=0}^{m-2}\left(\alpha_{k}^{2}\right)
\end{array}\right.
$$

where the first term $E\left(\overline{\mathbf{x}}^{\prime}, \mathbf{v}^{\prime}, \mathbf{x}\right)$ is the likelihood energy term and the second term $E(\mathbf{x})$ is the prior energy term, used to constrain the estimated shape to a realistic result. $\rho$ is a parameter that controls the relative weighting between these two terms. $\mathbf{p}_{k}(j)$ is the $j$ th tuple (a $3 \mathrm{D}$ vector) of the $k$ th shape basis eigenvector.

To determine $\left\{\alpha_{k}\right\}$, the cost function is differentiated with respect to the shape parameters and equated to zero resulting in a linear system of $(m-1)$ unknowns, which can then be solved with standard methods. ${ }^{61}$ To generate the surface model from the calculated shape parameters, I adopted a strategy that was introduced in Zheng et al., ${ }^{62}$ i.e., a cut-off point was set such that only the first few modes before the cut-off point and their corresponding shape parameters were used in the model instantiation. For all experiments reported in this paper, the cut-off point was chosen to be 10 . The reason for choosing this number is that because with the first 10 eigenmodes about $98.0 \%$ total amount of variations of the PDM is explained.

Regularized shape deformation In this stage, one needs to first set up the correspondences between the input images and a statistically instantiated surface model that is obtained through solving Eq. (6). To keep the same notation, let's assume that the image points in this stage are $\mathbf{v}^{\prime}=\left\{v_{i}^{\prime}=\left(x_{i}^{\prime}, y_{i}^{\prime}, z_{i}^{\prime}\right)\right.$; $i=0,1, \ldots, l-1\}$ and that the model points in this stage are $\mathbf{v}=\left\{v_{i}=\left(\mathbf{x}_{j}\right)_{i}=\left(x_{i}, y_{i}, z_{i}\right) ; \quad i=0,1, \ldots\right.$, $l-1\}$, where $l$ is the number of the matched point pairs and $\left(\mathbf{x}_{j}\right)_{i}$ means that the $j$ th model point $\mathbf{x}_{j}$ on the statistically instantiated model $\mathbf{x}$ is the closest point to the $i$ th image point $v_{i}^{\prime}$. The regularized shape deformation is described as a regression problem of finding a 3D spatial transform $\mathbf{t}: \Re^{3} \rightarrow \Re^{3}$ that minimizes following cost function, ${ }^{61,62}$

$$
E(\mathbf{t})=(1 / l) \cdot \sum_{i=0}^{l-1}\left\|v_{i}^{\prime}-\mathbf{t}\left(v_{i}\right)\right\|^{2}+\tau \cdot \frac{\log (m)}{\log (3 l)} \cdot L(\mathbf{t})
$$


where $\tau \geq 0$ is a parameter controlling the fitting quality and the regularization constraint; $m$ is the number of training surface models; $\mathbf{t}(\mathbf{v})=\left\{\mathbf{t}\left(v_{i}\right)\right\}$ are the results of applying the mapping on the model points and $L(\mathbf{t})$ is a thin-plate splines based regularization functional defined on the nonlinear mapping $\mathbf{t}$ and has following form, ${ }^{9}$

$$
\left\{\begin{aligned}
L(\mathbf{t})= & \iiint_{\Re^{3}}(B(\mathbf{t})) d x d y d z ; \quad \text { and } \\
B(\cdot)= & \left(\frac{\partial^{2}}{\partial x^{2}}\right)^{2}+2\left(\frac{\partial^{2}}{\partial x \partial y}\right)^{2}+\left(\frac{\partial^{2}}{\partial y^{2}}\right)^{2} \\
& +2\left(\frac{\partial^{2}}{\partial y \partial z}\right)^{2}+\left(\frac{\partial^{2}}{\partial z^{2}}\right)^{2}+2\left(\frac{\partial^{2}}{\partial z \partial x}\right)^{2}
\end{aligned}\right.
$$

For details about how to solve Eq. (7), I refer to previous work. ${ }^{61,62}$

\section{Performance Evaluation}

Post-operative X-ray radiographs and post-operative CT images of ten cadavers (20 hips) and five patients (none of them are part of the training populations) were used in a performance evaluation study. Two different X-ray machines were used to acquire the $\mathrm{X}$-ray radiographs. The $\mathrm{X}$-ray machine for all ten cadaveric pelvises were acquired by one $\mathrm{X}$-ray machine with a focal-point-to-film distance of $1200 \mathrm{~mm}$ and a pixel size of $0.143 \mathrm{~mm}$, while the $\mathrm{X}$-ray radiographs for five patients were acquired by the other X-ray machine with a focal-point-to-film distance of $1016 \mathrm{~mm}$ and a pixel size of $0.169 \mathrm{~mm}$.

The purpose of the cadaveric pelvis experiment was to quantitatively evaluate the accuracy and the robustness of the present approach. For this purpose, ten cadaveric pelvises and 20 all-polyethylene acetabular components were used. Two studies were performed. The first study was conducted on all ten cadaveric pelvises. For each cadaveric pelvis used in this study, a post-operative X-ray radiograph and a post-operative CT scan were acquired after the prostheses were implanted in both hips. All X-ray radiographs used in this study were acquired in a standardized way according to Widmer, who suggested in his work $^{57}$ that the coronal plane of the pelvis should be placed as parallel as possible to the X-ray film. The second study was performed on one cadaveric pelvis that was randomly chosen from the ten pelvises. In this study, besides the post-operative X-ray radiograph and the post-operative $\mathrm{CT}$ scan of the randomly chosen pelvis that were acquired in the first study, additionally three X-ray radiographs were acquired from non-standard positions.

A retrospective experiment involving clinical datasets of five patients (two males and three females) who underwent bilateral (but not simultaneous) primary THA was used to investigate the feasibility of using the present approach in clinical routine. Each patient dataset comprised of a post-operative X-ray radiograph and a post-operative CT scan.

The ground truth of each case was obtained from the associated post-operative CT images using the "HipMatch" program that was introduced in Zheng et al. ${ }^{65}$ It starts with the extraction of the APP from the post-operative CT images by interactively picking anatomical landmarks such as the left and the right ASIS and the left and the right pubic tubercles (this APP was named as "CT-based APP"). An anatomical coordinate system is then established using the four landmarks following the method introduced in Zheng et $a l .{ }^{64}$ Furthermore, to estimate the acetabular cup orientation, one needs to define the normal to the cup opening plane, which is done by interactively picking another three points from the post-operative CT images using the same program. ${ }^{65}$ The positions of these three points are controlled using the three orthogonal multi-planar reconstruction viewers as implemented in the "HipMatch" program. ${ }^{65}$ The ultimate goal is to make sure that all these three points fall on a plane that is parallel to the plane of the cup opening. As soon as all landmarks are extracted from the post-operative CT images, the cup orientation can be calculated. For details about how to use the "HipMatch" program to establish the ground truth for each case from its associated post-operative CT images, I refer to Zheng et ll. $^{65}$

According to Murray, ${ }^{39}$ acetabular cup anteversion and inclination can be defined in different ways. In this article, for both the ground truth measurement and the measurement generated from the present approach, I used the anatomical anteversion and the radiographic inclination that were calculated according to the definitions given by Murray. ${ }^{39}$ The reason why these particular angles are chosen is due to their wide usage in most of the commercial navigation systems. ${ }^{42}$ Thus, the radiological anteversion measured from the plain film using the technique described in Widmer ${ }^{57}$ was converted to the anatomical anteversion using a formula described by Murray ${ }^{39}$ for later comparison purpose.

Experiments on both cadaver and clinical datasets were conducted to evaluate the performance of the present approach and to compare the accuracy of the present approach to those of other methods. Specifically, for each dataset used in the first study of the cadaver experiment and in the patient experiment, acetabular cup orientation was measured using the present approach, the CT-based $2 \mathrm{D} / 3 \mathrm{D}$ rigid image registration approach that was described in Zheng et al., ${ }^{64,65}$ and the radiographic measurement method introduced in Widmer. ${ }^{57}$ These measurements were 
then compared to ground truths calculated from the associated post-operative CT images. All measurements were input to Microsoft Excel ${ }^{\circledR} 2007$ (Microsoft Corporation, Redmond, USA) for statistics. The significant level was chosen as $\alpha=0.01$.

The situation in the second study of the cadaver experiment was slightly different. Because the three additional X-ray radiographs of the randomly chosen cadaver are acquired from non-standard positions, Widmer's method cannot be applied directly any more. $^{57}$ To investigate the feasibility of using a 3D model reconstructed from a non-standard X-ray radiograph for estimating post-operative cup orientation, here I proposed to perform a surface-based scaled rigid registration between the model reconstructed from the non-standard X-ray radiograph and a model reconstructed from the standard X-ray radiograph of the randomly chosen pelvis. The transformed model could then be used together with the standard X-ray radiograph to estimate the post-operative cup orientation based on the present approach.

Furthermore, when the CT-based 2D/3D rigid image registration approach ${ }^{64,65}$ was used to compute the cup orientation for each case, the transformation that was obtained from the $2 \mathrm{D} / 3 \mathrm{D}$ rigid image registration allowed one to transform the CT-based APP from the CT data coordinate system to the X-ray image coordinate system for a computation of the pelvic tilt and rotation with respect to the X-ray imaging table/plate. According to Tannast et al., ${ }^{50}$ the pelvic tilt angle is defined as the difference between the anatomical coordinate system defined on the CT-based APP of the pelvis and the plane of the X-ray imaging table around the pelvic horizontal axis, while the pelvic rotation angle is defined as the difference between these two coordinate systems around the pelvic vertical axis. For details, I refer to Tannast et al. ${ }^{50}$

\section{RESULTS}

Results of the first study of the cadaveric pelvis experiment are presented in Tables 1, 2, and 3. In this study, the pelvic tilt and rotation angles of the ten cadaveric pelvis measured by the CT-based $2 \mathrm{D} / 3 \mathrm{D}$ rigid image registration method ${ }^{64,65}$ are shown in Table 1. A mean tilt angle of $3.8 \pm 6.5^{\circ}$ and a mean rotation angle of $-0.4 \pm 1.5^{\circ}$ were found, which

TABLE 1. The pelvic tilt and rotation angles of the ten cadaveric pelvises measured by the CT-based 2D/3D rigid image registration method. ${ }^{6}$

\begin{tabular}{|c|c|c|c|c|c|c|c|c|c|c|c|}
\hline Cadavers & $\# 1$ & \#2 & \#3 & $\# 4$ & $\# 5$ & $\# 6$ & \#7 & $\# 8$ & $\# 9$ & $\# 10$ & Mean \pm STD \\
\hline Tilt $\left(^{\circ}\right)$ & 10.3 & -8.6 & -0.1 & 5.7 & 8.5 & 9.1 & -5.7 & 4 & 7.9 & 6.4 & $3.8 \pm 6.5$ \\
\hline Rotation $\left(^{\circ}\right)$ & 0.1 & -1.4 & -0.2 & -2.9 & -0.9 & -1.9 & -0.1 & 0.5 & -0.1 & 2.7 & $-0.4 \pm 1.5$ \\
\hline
\end{tabular}

TABLE 2. Absolute estimation errors when different methods were used to measure the cup orientations $(n=20)$.

\begin{tabular}{|c|c|c|c|}
\hline Angle & Mean $\pm \operatorname{STD}\left({ }^{\circ}\right)$ & Minimum Error $\left({ }^{\circ}\right)$ & Maximum Error $\left({ }^{\circ}\right)$ \\
\hline \multicolumn{4}{|c|}{ Absolute estimation errors when the radiographic measurement method ${ }^{57}$ was used } \\
\hline Inclination & $2.5 \pm 2.5$ & 0.3 & 8.0 \\
\hline Anteversion & $5.6 \pm 3.6$ & 1.0 & 11.4 \\
\hline \multicolumn{4}{|c|}{ Absolute estimation errors when the present approach was used } \\
\hline Inclination & $1.5 \pm 1.5$ & 0.1 & 5.5 \\
\hline Anteversion & $1.7 \pm 1.4$ & 0.1 & 5.0 \\
\hline \multicolumn{4}{|c|}{ Absolute estimation errors when the CT-based 2D/3D registration method ${ }^{64,65}$ was used } \\
\hline Inclination & $1.0 \pm 0.6$ & 0.0 & 2.1 \\
\hline Anteversion & $1.1 \pm 0.9$ & 0.1 & 2.5 \\
\hline
\end{tabular}

TABLE 3. The paired two-tailed $t$-test results when the errors of different methods were compared to each other $(n=20)$.

\begin{tabular}{lcc}
\hline & $\begin{array}{c}\text { Errors of the present } \\
\text { approach vs. Errors } \\
\text { of the radiographic } \\
\text { measurement method }\end{array}$ & $\begin{array}{c}\text { Errors of the present } \\
\text { approach vs. Errors of the CT-based } \\
\text { 2D/3D registration approach }\end{array}$ \\
\hline $\begin{array}{l}\text {-Value of inclination observation } \\
p \text {-Value of anteversion observation }\end{array}$ & $0.11>0.01$ & $0.05>0.01$ \\
$0.0003<0.01$ & $0.10>0.01$ \\
\hline
\end{tabular}


confirmed that all radiographs used in this study were acquired in a standardized way. Taking the measurements obtained from the post-operative CT images as the ground truths, the absolute errors when different approaches were used to measure the post-operative cup orientations are presented in Table 2. When the radiographic measurement method as introduced in Widmer $^{57}$ was used, errors of $5.6 \pm 3.6^{\circ}$ were found for the anteversion and errors of $2.5 \pm 2.5^{\circ}$ were found for the inclination (see Table 2 for details). When the present approach was used, the errors were changed to $1.7 \pm 1.4^{\circ}$ for anteversion and $1.5 \pm 1.5^{\circ}$ for inclination (see Table 2 for details). When the CT-based 2D/ $3 \mathrm{D}$ rigid image registration approach ${ }^{64,65}$ was used, errors of $1.1 \pm 0.9^{\circ}$ were found for the anteversion and errors of $1.0 \pm 0.6^{\circ}$ were found for the inclination (see Table 2 for details), which were slightly better than the results obtained with the present approach. When the measurement results obtained by these three approaches were compared to each other, it was found that the measurement results obtained by the CT-based 2D/3D registration approach ${ }^{64,65}$ had the smallest average errors for both the anteversion and the inclination and that the measurement results obtained by the method introduced in Widmer ${ }^{57}$ had the largest average errors for both the anteversion and the inclination. To determine whether the differences are statistically significant, a paired two-tailed t-test was used. Table 3 shows the test results. It was found that the improvement of the present approach over the radiograph-based method ${ }^{57}$ on measuring the postoperative cup anteversion was statistically significant $(p=0.0003<0.01)$, while the improvement of the present approach over the radiograph-based method $^{57}$ on measuring the post-operative cup inclination was not $(p=0.11>0.01)$. When the results obtained by

TABLE 4. The tilt and rotation angles of the pelvic at each image acquisition position measured by the CT-based 2D/3D rigid image registration approach. ${ }^{64,65}$

\begin{tabular}{lrrrrr} 
Images & Image \#1 & Image \#2 & Image \#3 & Image \#4 & \multicolumn{1}{c}{ Average } \\
\hline Tilt $\left(^{\circ}\right)$ & -8.6 & -39.3 & -13.3 & 1.8 & $-14.9 \pm 17.5$ \\
Rotation & -1.4 & 0.1 & 27.8 & -29.0 & $-0.6 \pm 23.2$ \\
$\left(^{\circ}\right)$ & & & & & \\
\hline
\end{tabular}

the present approach were compared to those obtained by the CT-based $2 \mathrm{D} / 3 \mathrm{D}$ registration approach, ${ }^{64,65}$ it was found that the differences between these two measurement results were not statistically significant for both the anteversion $(p=0.10>0.01)$ and the inclination $(p=0.05>0.01)$.

Results of the second study of the cadaveric pelvis experiment are described in Tables 4 and 5. This study was performed on the datasets of the randomly chosen cadaver pelvis, i.e., Cadaver \#2. Table 4 shows the tilt and rotation angles of the pelvis at each image acquisition position. They were measured by the CT-based 2D/3D rigid image registration method. ${ }^{64,65}$ A mean tilt angle of $-14.9 \pm 17.5^{\circ}$ and a mean rotation angle of $-0.6 \pm 23.2^{\circ}$ were found. Although three of the four X-ray radiographs of the pelvis were acquired from non-standard positions, the present $2 \mathrm{D} / 3 \mathrm{D}$ reconstruction approach could successfully reconstruct a scaled, patient-specific 3D model from each image. Figure 8 shows the four X-ray radiographs (Fig. 8a) and screenshots of the corresponding $2 \mathrm{D} / 3 \mathrm{D}$ reconstruction results (Fig. 8b). After a model reconstructed from one of the non-standard X-ray radiograph was matched to the model reconstructed from the standard $\mathrm{X}$-ray radiograph, it could be used together with the standard X-ray radiograph (Image \#1) to measure the cup orientation based on the present approach. The absolute errors on estimating the cup orientation based on the reconstructed 3D models are presented in Table 5. Errors of $2.5 \pm 1.2^{\circ}$ were found for the anteversion and errors of $1.4 \pm 1.1^{\circ}$ were found for the inclination. The results on measuring the cup anteversion in this study were slightly less accurate than those obtained in the first study, where only the X-ray radiographs acquired from the standard positions were used.

Results of the patients' pelvis experiment are presented in Tables 6 and 7. The tilt and rotation angles of each patient's pelvis measured by the CT-based $2 \mathrm{D} / 3 \mathrm{D}$ rigid image registration approach ${ }^{64,65}$ are shown in Table 6. A mean tilt angle of $-10.6 \pm 14.1^{\circ}$ and a mean rotation angle of $0.1 \pm 0.9^{\circ}$ were found, which demonstrated that although surgeons were asked to place the coronal plane of each patient's pelvis to be as parallel as possible to the X-ray film, it was difficult,

TABLE 5. Absolute errors on estimating the cup orientation based on one of the reconstructed 3D models and the standard X-ray radiograph (Image \#1).

\begin{tabular}{|c|c|c|c|c|c|c|c|c|c|}
\hline \multirow{2}{*}{$\begin{array}{l}\text { Reconstructed model } \\
\text { Side }\end{array}$} & \multicolumn{2}{|c|}{$\begin{array}{c}\text { Model from } \\
\text { image \#1 }\end{array}$} & \multicolumn{2}{|c|}{$\begin{array}{c}\text { Model from } \\
\text { image \#2 }\end{array}$} & \multicolumn{2}{|c|}{$\begin{array}{c}\text { Model from } \\
\text { image \#3 }\end{array}$} & \multicolumn{2}{|c|}{$\begin{array}{c}\text { Model from } \\
\text { image \#3 }\end{array}$} & \multirow{2}{*}{$\begin{array}{l}\text { Average } \\
\text { Not Applied }\end{array}$} \\
\hline & Left & Right & Left & Right & Left & Right & Left & Right & \\
\hline Inclination $\left(^{\circ}\right)$ & 0.9 & 0.1 & 1.9 & 1.3 & 3.3 & 2.2 & 0.3 & 1.1 & $1.4 \pm 1.1$ \\
\hline Anteversion $\left(^{\circ}\right)$ & 3.7 & 1.2 & 3.9 & 3.8 & 2.4 & 0.9 & 1.6 & 2.5 & $2.5 \pm 1.2$ \\
\hline
\end{tabular}


(a)
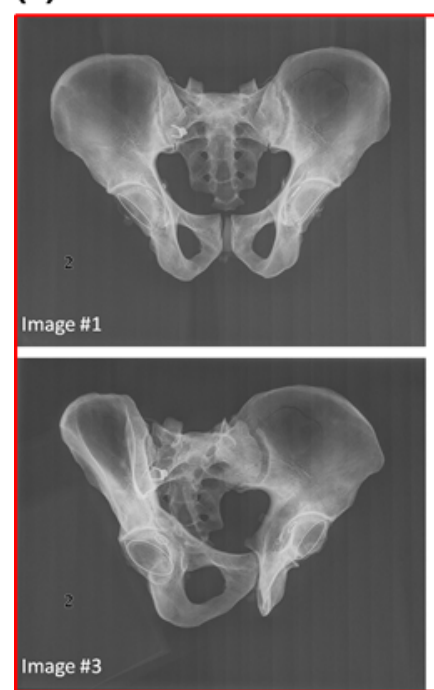

(b)
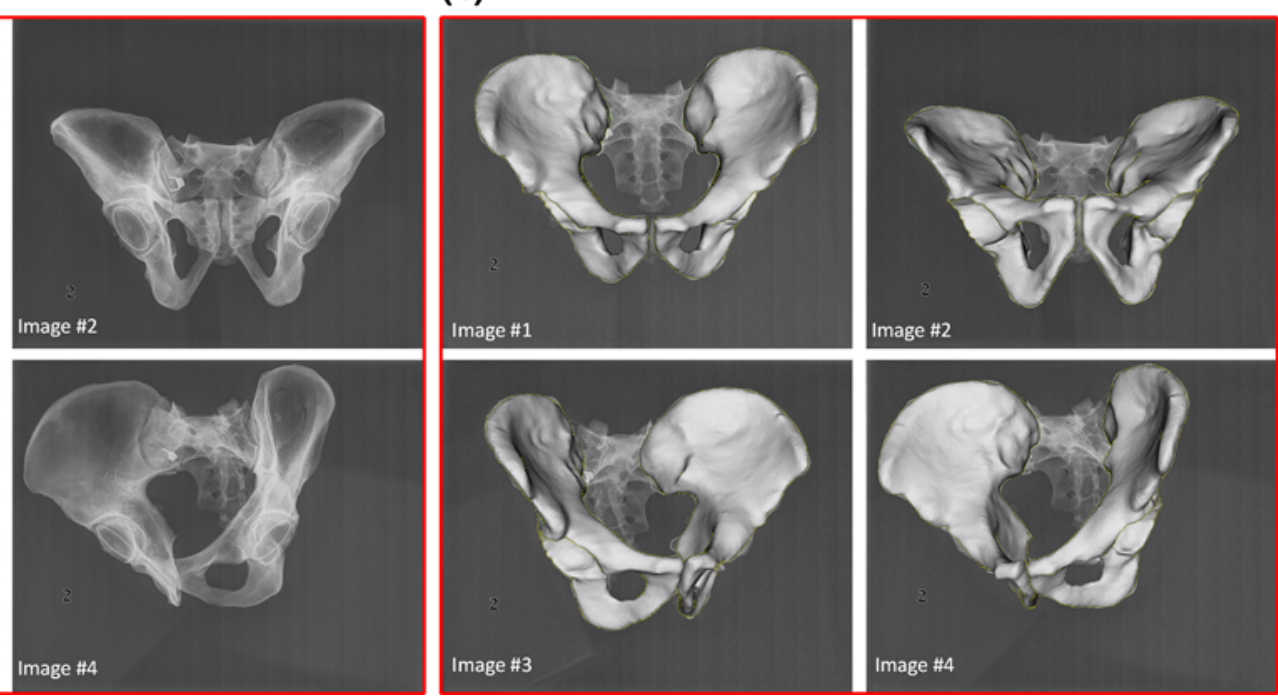

FIGURE 8. X-ray radiographs acquired from both standard and non-standard positions, and the corresponding 2D/3D reconstruction results. (a) The four X-ray radiographs used in the second study of the cadaver experiment. Except the first image that was acquired in a standard position, all other three images were acquired from non-standard positions; and (b) the corresponding 2D/3D reconstruction results.

TABLE 6. The tilt and rotation angles of each patient's pelvis measured by the CT-based 2D/3D rigid image registration approach. ${ }^{64,65}$

\begin{tabular}{lcccccc}
\hline Patients & $\begin{array}{c}\text { Male_01, } \\
\text { right cup }\end{array}$ & $\begin{array}{c}\text { Female_01, } \\
\text { left cup }\end{array}$ & $\begin{array}{c}\text { Female_02, } \\
\text { right cup }\end{array}$ & $\begin{array}{c}\text { Female_03, } \\
\text { Right cup }\end{array}$ & $\begin{array}{c}\text { Male_02, } \\
\text { Right cup }\end{array}$ & Average \\
\hline Tilt $\left(^{\circ}\right)$ & -25.0 & -16.6 & 1.8 & -20.0 & 6.9 & $-10.6 \pm 14.1$ \\
Rotation $\left(^{\circ}\right)$ & 1.1 & 0.0 & 0.1 & 0.8 & -1.3 & $0.1 \pm 0.9$ \\
\hline
\end{tabular}

TABLE 7. Absolute estimation errors when different methods were used to measure the cup orientations.

\begin{tabular}{|c|c|c|c|c|c|c|}
\hline Patients & $\begin{array}{l}\text { Male_01, } \\
\text { right cup }\end{array}$ & $\begin{array}{l}\text { Female_01, } \\
\text { left cup }\end{array}$ & $\begin{array}{l}\text { Female_02, } \\
\text { right cup }\end{array}$ & $\begin{array}{c}\text { Female_03, } \\
\text { Right cup }\end{array}$ & $\begin{array}{l}\text { Male_02, } \\
\text { Right cup }\end{array}$ & Average \\
\hline \multicolumn{7}{|c|}{ Estimation errors when the radiographic measurement method ${ }^{57}$ was used } \\
\hline Inclination $\left({ }^{\circ}\right)$ & 0.4 & 4.6 & 2.1 & 1.6 & 4.1 & $2.6 \pm 1.8$ \\
\hline Anteversion $\left({ }^{\circ}\right)$ & 0.4 & 8.1 & 4.9 & 4.3 & 3.3 & $4.2 \pm 2.8$ \\
\hline \multicolumn{7}{|c|}{ Estimation errors when the present approach was used } \\
\hline Inclination $\left({ }^{\circ}\right)$ & 0.8 & 2.9 & 2.0 & 2.2 & 2.0 & $2.0 \pm 0.8$ \\
\hline Anteversion $\left({ }^{\circ}\right)$ & 1.5 & 1.7 & 4.4 & 2.2 & 1.2 & $2.2 \pm 1.3$ \\
\hline \multicolumn{7}{|c|}{ Estimation errors when the CT-based 2D/3D registration approach was used ${ }^{64,65}$} \\
\hline Inclination $\left({ }^{\circ}\right)$ & 0.8 & 0.7 & 0.7 & 1.2 & 1.8 & $1.0 \pm 0.5$ \\
\hline Anteversion $\left({ }^{\circ}\right)$ & 0.5 & 1.2 & 2.5 & 0.8 & 1.3 & $1.3 \pm 0.8$ \\
\hline
\end{tabular}

if it was not impossible, to control the exact orientation of the pelvis, specially the pelvic tilt angle. Taking the measurements obtained from the post-operative CT images as the ground truths, the absolute errors when different approaches were used to measure the postoperative cup orientations are presented in Table 7. When the radiograph-based method ${ }^{57}$ was used, errors of $4.2 \pm 2.8^{\circ}$ were found for the anteversion and errors of $2.6 \pm 1.8^{\circ}$ were found for the inclination. When the results measured by the present approach were compared to their associated ground truths, differences of $2.2 \pm 1.3^{\circ}$ were found for anteversion and differences of $2.0 \pm 0.8^{\circ}$ were found for the inclination. When the CT-based 2D/3D registration approach ${ }^{64,65}$ was used, the differences were reduced to $1.3 \pm 0.8^{\circ}$ for anteversion and $1.0 \pm 0.5^{\circ}$ for inclination, respectively. 


\section{DISCUSSIONS}

Accurate assessment of the cup orientation is important for evaluation of outcome after THA, but the inability to measure cup orientation accurately on standard AP X-ray radiographs limits one's ability to determine optimal cup orientations, to assess new treatment methods for improved cup placement during surgery, and to correlate the cup orientation to osteolysis, wear, and instability. Therefore, the goal of the present study is to develop and to validate a novel statistically deformable $2 \mathrm{D} / 3 \mathrm{D}$ registration approach in order to calculate post-operative cup orientation from a single standard AP X-ray radiograph via a patient-specific 3D model of the pelvis. The results of the experiments that were conducted on datasets of ten cadavers (20 hips) and five patients demonstrated that the present technique was apparently more accurate than the simple radiographic measurement method, especially on measuring cup anteversion, where a statistically significant difference was found. Compared to the CT-based 2D/3D registration approach, ${ }^{64,65}$ the present technique was slightly less accurate but the differences were not statistically significant.

The advantages and the disadvantages of the four different methods that are used nowadays to measure the post-operative cup orientation are summarized in Table 8. From this table, one can see that there are several advantages of the present technique over other methods for measuring the post-operative cup orientation. Several studies, $2,22,33,42,49,64,65$ including the one presented in this article, have shown that the radiographic measurement methods, though easy to use, cannot produce accurate results, especially for the anteversion measurements, due to the wide variability in individual pelvic orientation relative to X-ray plate during image acquisition. Previous studies ${ }^{42,64}$ reported that the errors in measuring the cup anteversions with the radiographic measurement method could be as high as $20^{\circ}$. On the other side, post-operative CT-based methods, which are regarded as the most reliable methods for measuring post-operative cup orientation,,$^{2,4,22,31,33}$ are not widely be used in clinical routine because they require not only the additional expense but also the additional radiation doses to the patient. The recently introduced CT-based $2 \mathrm{D} / 3 \mathrm{D}$ rigid image registration methods $8,20,28,42,64,65$ have shown reasonably accurate results in calculation of post-operative cup orientation. But again, their extensive usage in clinical routine is still limited, largely due to the requirement of having a CT study of the patient at some point during treatment, which brings in the radiation issue. Furthermore, it is questionable whether the CT would be available for vast majority of THA procedures performed nowadays. ${ }^{42}$ In contrast, the present technique only requires a single standard post-operative AP X-ray radiograph to reconstruct the patient-specific surface model of the pelvis and to calculate the post-operative cup orientation. No CT study of the patient is needed any more, which eliminates the radiation issue. This has a clear medical and also economical advantage in the clinical setting.

As a limitation of the present study, one has to discuss taking the one-time post-operative CT-based measurement as the ground truth. Previously, several studies $^{2,4,22,31,33}$ have suggested that CT-based methods seem to be the most reliable method for noninvasive post-operative assessment of the acetabular cup orientation with experienced and trained observers. Nevertheless, intra- and inter-observer variability is still present. ${ }^{31}$ However, the influence of such variability on the results could not be determined with the present study setup.

It is worth noting that a scaled, patient-specific surface model can be reconstructed by the present approach from a single conventional X-ray radiograph, which was previously regarded by Metz and Fencil $^{35}$ as an imaging method that does not provide information concerning the object's 3D structure. The reasons why the present approach works could be explained briefly as follows. Although all the edge points used in the $2 \mathrm{D} / 3 \mathrm{D}$ reconstruction are taken from one imaging plane such that they do not contain any depth information along the projection direction, the image-to-model correspondence establishing process in the present approach, however, converts those planar $2 \mathrm{D}$ edge points to a set of $3 \mathrm{D}$ points with different depths along the projection direction (see Fig. 7 for an illustration). The sophisticated $2 \mathrm{D} / 3 \mathrm{D}$ reconstruction technique as presented in this article then iteratively alternates the correspondence establishing and the parameter optimization procedures (more specifically, at different stages, different parameters are optimized: affine transformation parameters in the scaled rigid registration stage, statistical shape coefficient parameters in the statistical instantiation stage and the nonlinear shape deformation parameters in the regularized shape deformation stage) such that a patient-specific surface model can be reconstructed from the single $\mathrm{X}$-ray radiograph up to an unknown scale factor. For the application reported in this article, not knowing the exact scale factor is not an important issue because only cup orientation is the interest. The results of the postoperative cup orientation measurement experiments that were conducted on datasets of ten cadavers $(20$ hips) and five patients confirmed this conclusion. A more careful look of the three-stage reconstruction technique shows that at each stage the exact depth assigned to each selected edge point is dependent on the image-to-model correspondence establishing process 
TABLE 8. Advantages and disadvantages when different methods were used to estimate post-operative cup orientation.

\begin{tabular}{|c|c|c|}
\hline Methods & Advantages & Disadvantages \\
\hline $\begin{array}{l}\text { Post-operative CT images-based } \\
\text { method }\end{array}$ & $\begin{array}{l}\text { The most accurate method among all } \\
\text { four methods }\end{array}$ & $\begin{array}{l}\text { CT-acquisition is not part of the standard } \\
\text { diagnostics and treatment protocol for } \\
\text { every patient. Furthermore, for a } \\
\text { long-term follow-up, one has to } \\
\text { acquire many CT scans, which involve } \\
\text { additional cost and radiations to the } \\
\text { patient as well as additional logistic } \\
\text { effort to the clinics. }\end{array}$ \\
\hline $\begin{array}{l}\text { CT-based } 2 D / 3 D \text { rigid image } \\
\text { registration method }\end{array}$ & $\begin{array}{l}\text { Less accurate than the post-operative } \\
\text { CT images-based method but more } \\
\text { accurate than other two methods; } \\
\text { Furthermore, it only needs a CT study } \\
\text { of the patient at some point during the } \\
\text { treatment }\end{array}$ & $\begin{array}{l}\text { CT-acquisition is not part of the standard } \\
\text { diagnostics and treatment protocol for } \\
\text { every patient. It can be applied to a } \\
\text { long-term follow-up as long as a CT } \\
\text { study of the patient at some point } \\
\text { during the treatment is available. } \\
\text { However, it cannot be applied to the } \\
\text { situations such as retrospective } \\
\text { studies or studies involving an earlier } \\
\text { cup design when there is no CT study } \\
\text { available. }\end{array}$ \\
\hline The radiograph-based method & $\begin{array}{l}\text { Radiograph acquisition is part of the } \\
\text { standard diagnostics and treatment } \\
\text { protocol. It is used routinely because } \\
\text { of its simplicity, availability, and } \\
\text { minimal expense associated with its } \\
\text { acquisition }\end{array}$ & $\begin{array}{l}\text { The least accurate method among all } \\
\text { four methods. Several stud- } \\
\text { ies, }{ }^{2,22,33,42,49,64,65} \text { including the one } \\
\text { presented in this article, demonstrate } \\
\text { that the radiograph-based method } \\
\text { cannot produce accurate results, } \\
\text { especially for the anteversion } \\
\text { measurement. Previous studies }{ }^{42,64} \\
\text { reported that the errors in measuring } \\
\text { the cup anteversion with the radio- } \\
\text { graph-based method could be as high } \\
\text { as } 20^{\circ} \text {. }\end{array}$ \\
\hline The present approach & $\begin{array}{l}\text { No CT study of the patient is required for } \\
\text { a true 3D measurement of the post- } \\
\text { operative cup orientation. It has the } \\
\text { same advantage as the radiograph- } \\
\text { based method but with much higher } \\
\text { measurement accuracy. Thus, it can } \\
\text { be applied to all kinds of situations. } \\
\text { Furthermore, the scaled, patient- } \\
\text { specific 3D model derived from the } \\
\text { single X-ray image can play an } \\
\text { important role in planning and } \\
\text { supporting various computer assisted } \\
\text { surgical procedures }\end{array}$ & $\begin{array}{l}\text { Slightly less accurate than the post- } \\
\text { operative CT images-based method } \\
\text { and the CT-based 2D/3D rigid image } \\
\text { registration method. It also requires } \\
\text { the clinician to interactively define } \\
\text { the contours from the input X-ray } \\
\text { image. This procedure normally } \\
\text { takes two to four minutes. }\end{array}$ \\
\hline
\end{tabular}

as well as on the prior model that is used in the process, i.e., the mean model of the PDM in the first two stages or a surface model statistically instantiated from the PDM in the last stage. If one neglects the scaling effect between the assigned depths and the associated true depths, one can say to a certain extent that the PDM encodes the depth information for each selected point in the X-ray radiograph. Furthermore, the surface model obtained after the statistical instantiation stage contains more patient-specific information than the mean model of the PDM such that it allows for a more accurate depth assignment. This is also the reason why unlike previous attempts to instantiate a surface mode from a statistical shape model, ${ }^{5,6,16,24,25,32,45}$ one additional regularized shape deformation stage is added to the statistical instantiation to further refine the statistically instantiated surface model. Thus, the ability of the present approach in determining a scaled, patient-specific 3D surface model from a single projection image is, to a large extent, attributed to the combination of the statistical shape model, which contains the prior knowledge about the underlying 3D structure, with the sophisticated $2 \mathrm{D} / 3 \mathrm{D}$ reconstruction technique.

The differences between the present technique and other published work ${ }^{21,25,36,45}$ on reconstructing a 
patient-specific surface model of the pelvis from X-ray image(s) should be discussed. To the best knowledge of the author, this may be the first work on using a scaled, patient-specific surface model of the pelvis derived from a single standard AP X-ray radiograph for estimating the post-operative cup orientation. In contrast, previous studies on reconstructing a patient-specific surface model of the pelvis focus on evaluating the overall surface model reconstruction accuracy ${ }^{21,25,36,45}$ and/or on evaluating the $3 \mathrm{D}$ morphometry reconstruction accuracy. ${ }^{36}$ Both the work presented by Sadowsky et $a l^{45}$ and the work presented by Lamecker et al. ${ }^{25}$ use a SSM of the pelvis and are only evaluated by performing leave-one-out studies on simulated images, whose projection parameters are assumed to be known. When three simulated C-arm views were used, Sadowsky et al. ${ }^{45}$ reported a mean accuracy of $2.0 \mathrm{~mm}$. An intensity-based deformable $2 \mathrm{D} / 3 \mathrm{D}$ registration was used in their paper to match a SSM of the pelvis with the input simulated $\mathrm{C}$-arm views, which might be timeconsuming. Using two simulated $\mathrm{X}$-ray radiographs in a leave-one-out study, Lamecker et al. ${ }^{25}$ reported a mean accuracy of $2.4 \mathrm{~mm}$. However, their method asks for a clear contour identification from the AP and the lateral-medial (LM) X-ray radiographs. Although it is possible to identify contours from the simulated LM $\mathrm{X}$-ray radiograph where no surround soft tissues are presented, it will be definitely a challenge to identify contours from a real LM X-ray radiograph of a patient in a clinical setup, due to the poor image quality caused by superimposition of left and right pelvic structures with the surrounding soft tissues. The same challenge can also be posted to the method presented by Mitton et al., ${ }^{36}$ where 2D anatomical points and contours interactively identified from bi-planar radiography are used in a kriging method to deform a generic surface model, which in the best case scenario is equivalent to the mean model of a SSM. Recently, Kadoury et al. ${ }^{21}$ presented a kriging method for reconstructing a surface model of the pelvis from uncalibrated bi-planar X-ray images. However, the accuracy of their method depends on the accuracy of a manual landmark identification process, as they use a self-calibration algorithm that is based on the minimization of landmark re-projection errors. In contrast, the present approach only uses the anatomical landmarks to estimate an initial scaled rigid transformation. The exact rigid and non-rigid transformations between the image contours and the SSM of the pelvis are estimated through an iterative optimization procedure.

While accurate, the present approach has limitations. Although the present approach is applicable to all types of bearing, the precondition of the implant that can be measured by the present approach should have a circular opening surface of the acetabular cup.
The second limitation is due to the fact that Widmer's method $^{57}$ is used to calculate the cup radiographic version from the plain radiograph. The assumption behind Widmer's method ${ }^{57}$ is that the X-ray radiograph should be acquired in a standardized way. As demonstrated by the experimental results, although the $2 \mathrm{D} / 3 \mathrm{D}$ reconstruction approach can reconstruct a scaled, patient-specific model even from an X-ray radiograph acquired in a non-standard position, the present approach cannot be used directly to measure the post-operative cup orientation from the non-standard X-ray radiograph. Nevertheless, such a limitation can be addressed either by correcting radiographic cup version using the method presented by Tannast et al. ${ }^{51}$ or by performing a rigid $2 \mathrm{D} / 3 \mathrm{D}$ registration between the CAD model of the cup and the X-ray radiograph as done in the references. ${ }^{8,20,42}$ The third limitation is that the present approach in its current form only measures the acetabular cup orientation. Thus, more work needs to be done to support the combined anteversion technique. ${ }^{13}$ The last limitation discussed here is related with the number of training models used to construct the statistical shape model and the number of cases used to validate the present approach. From above analysis on how the present approach works, one can conclude that the scaled reconstruction accuracy is mainly dependent on the depth assignment accuracy. The accuracy of such an assignment, however, depends not only upon how accurate the imageto-model correspondences can be established but also upon how well the unknown, patient-specific shape variation can be covered by the statistical shape model that is constructed from a fixed number of training models. Although the image-to-model correspondence establishing process has been thoroughly validated in the previous works ${ }^{59,60,62,63}$ as well as in the present work, the statistical shape model used in the present study was only constructed from 14 pelvises with mixed gender. Furthermore, the validation of the present approach, though successful, was only conducted on datasets of ten cadaver pelvises and five patient pelvises. Thus, the results reported in this article are regarded still preliminary and more thorough validation study is needed before it can be transferred to clinical routine usage. Nonetheless, the experiment results from the present study demonstrate the efficacy of the present approach and the prediction power of the present approach can be enhanced in the future by incorporating more training models into the statistical shape model and/or by constructing a patient-oriented statistical shape model. One of the future directions that are worth to explore is to investigate whether a patient-oriented statistical shape model (e.g., a patient's gender-oriented statistical shape model) will improve the accuracy of the present approach. 


\section{SUMMARY AND CONCLUSIONS}

In summary, this article presents a statistically deformable $2 \mathrm{D} / 3 \mathrm{D}$ registration approach for estimating post-operative cup orientation from a single standard AP X-ray radiograph. When compared to the existing work, ${ }^{8,20,28,42,64,65}$ among the advantages such as the independence of a proprietary CAD model of the prosthesis and the elimination of a radiographspecific calibration, the most significant advantage of the present approach is the elimination of the necessity of possessing a CT study of the patient for an accurate measurement of the post-operative cup orientation. Thus, the technique presented in this article is more appropriate for long-term retrospective studies. The results of the preliminary experiments, performed on datasets of ten cadavers (20 hips) and of five patients, though preliminary, demonstrated its accuracy and the reliability. Thus, it is expected that the present technique, when thoroughly validated, can be used as a valuable tool to provide evidence-based information for evaluating surgical technologies (e.g., surgical navigation) and implant design. Furthermore, patientspecific surface models play important roles in planning and supporting various computer assisted surgical procedures. The present technique requires only one AP pelvic X-ray radiograph, which is part of the standard diagnostics and treatment loop in clinical routine, ${ }^{12,15}$ to derive a scaled, patient-specific surface models of the pelvis. Besides the application to the estimation of the post-operative cup orientation, the $2 \mathrm{D} / 3 \mathrm{D}$ reconstruction approach as presented in this article holds the potential to open ways for more costeffective planning and treatment of pelvic diseases.

\section{ACKNOWLEDGMENTS}

The author gratefully acknowledges the financial support from the Swiss National Centers of Competence in Research CO-ME. The author would also like to thank the associated editor and the anonymous reviewers for their constructive comments and suggestions.

\section{REFERENCES}

\footnotetext{
${ }^{1}$ Ali Kahn, M. A., P. H. Brakenbury, and I. S. Reynolds. Dislocation following total hip replacement. J. Bone Joint Surg. Br. 63B(2):214-218, 1981.

${ }^{2}$ Arai, N., S. Nakamura, T. Matsushita, and S. Suzuki. Minimal radiation dose computed tomography for measurement of cup orientation in total hip arthroplasty.
}

J. Arthroplasty, 2009 (in press). doi:10.1016/j.arth.2009. 01.020 .

${ }^{3}$ Bader, R. J., E. Steinhauser, G. Willmann, and R. Gradinger. The effects of implant position, design and wear on the range of motion after total hip arthroplasty. Hip Int. 11(2):80-90, 2001.

${ }^{4}$ Beckmann, J., C. Lüring, M. Tingart, S. Anders, J. Grifka, and F. X. Köck. Cup positioning in THA: current status and pitfalls. A systematic evaluation of the literature. Arch. Othop. Trauma Surg. 129:863-872, 2009.

${ }^{5}$ Benameur, S., M. Mignotte, S. Parent, H. Labelle, W. Skalli, and J. A. De Guise. 3D/2D registration and segmentation of scoliotic vertebra using statistical models. Comput. Med. Imaging Graph. 27:321-337, 2003.

${ }^{6}$ Benameur, S., M. Mignotte, S. Parent, H. Labelle, W. Skalli, and J. A. De Guise. A hierarchical statistical modeling approach for the unsupervised 3-D reconstruction of the scoliotic spine. IEEE Trans. Biomed. Eng. 52:2041-2057, 2005.

${ }^{7}$ Besl, P., and N. D. McKay. A method for registration of 3D shapes. IEEE Trans. Pattern Anal. Mach. Intell. 14:239-256, 1992.

${ }^{8}$ Blendea, S., K. Eckman, B. Jaramaz, T. J. Levison, and A. M. DiGioia, III. Measurements of acetabular cup position and pelvic spatial orientation after total hip arthroplasty using computed tomography/radiography matching. Comput. Aided Surg. 10:37-43, 2005.

${ }^{9}$ Bookstein, F. Principal warps: thin-plate splines and the decomposition of deformations. IEEE Trans. Pattern Anal. Mach. Intell. 11:567-585, 1989.

${ }^{10}$ Cootes, T. F., A. Hill, C. J. Taylor, and J. Haslam. The use of active shape models for locating structures in medical images. Image Vis. Comput. 12:355-366, 1994.

${ }^{11}$ Cootes, T. F., C. J. Taylor, D. H. Cooper, and J. Graham. Active shape models-their training and application. Comput. Vis. Image Underst. 61:38-59, 1995.

${ }^{12}$ Della Valle, C. J., K. Kaplan, A. Jazrawi, S. Ahmed, and W. L. Jaffe. Primary total hip arthroplasty with a flanged, cemented all-polyethylene acetabular component: evaluation at a minimum of 20 years. J. Arthroplasty 19:23-26, 2004.

${ }^{13}$ Dorr, L. D., A. Malik, M. Dastane, and Z. Wan. Combined anteversion technique for total hip arthroplasty. Clin. Orthop. Relat. Res. 467:119-127, 2009.

${ }^{14}$ Dryden, I., and K. Mardia. Statistical Shape Analysis. Wiley Series in Probability and Statistics. New York: Jonn Wiley \& Sons, Inc., 1998.

${ }^{15}$ Eggli, S., M. Pisan, and M. E. Müller. The value of preoperative planning for total hip arthroplasty. The Journal of Bone and Joint Surgery 80:382-390, 1998.

${ }^{16}$ Fleute, M., and S. Lavallée. Nonrigid $3 \mathrm{D} / 2 \mathrm{D}$ registration of images using statistical models. In: Proceedings of the 1998 International Conference on Medical Image Computing and Computer-assisted Intervention (MICCAI 1998), LNCS 1496:138-147, Springer 1998.

${ }^{17}$ Hertzmann, A., and D. Zorin. Illustrating smooth surfaces. In: Proceedings of the 27th Annual Conference on Computer Graphics and Interactive Techniques (SIGGRAPH'00). New York: ACM Press/Addison-Wesley Publishing Co., 2000, pp. 517-526.

${ }^{18}$ Humbert, L., J. A. De Guise, B. Aubert, B. Godbout, and W. Skalli. 3D Reconstruction of the spine from biplanar X-rays using parametric models based on transversal and longitudinal inferences. Med. Eng. Phys. 31:681-687, 2009. 
${ }^{19}$ Jaramaz, B., A. M. DiGioia III, M. Blackwell, and C. Nikou. Computer assisted measurement of cup placement in total hip replacement. Clin. Orthop. Relat. Res. 354:70-81, 1998.

${ }^{20}$ Jaramaz, B., and K. Eckman. 2D/3D registration for measurement of implant alignment after total hip replacement. In: Proceedings of the 9th International Conference on Medical Image Computing and Computer-assisted Intervention (MICCAI 2006). LNCS 4191:653-661, 2006.

${ }^{21}$ Kadoury, S., F. Cheriet, J. Dansereau, and H. Labelle. Three-dimensional reconstruction of the scoliotic spine and pelvis from uncalibrated biplanar X-ray images. J. Spinal Disord. Tech. 20:160-167, 2007.

${ }^{22}$ Kalteis, T., M. Handel, T. Herold, L. Perlick, C. Paetzel, and J. Grifka. Position of the acetabular cup-accuracy of radiographic calculation compared to CT-based measurement. Eur. J. Radiol. 58:294-300, 2006.

${ }^{23}$ Kendall, D. A survey of the statistical theory of shape. Stat. Sci. 4:87-120, 1989.

${ }^{24}$ Kurazume, R., K. Nakamura, T. Okada, Y. Sato, N. Sugano, T. Koyama, Y. Iwashita, and T. Hasegawa. 3D reconstruction of a femoral shape using a parametric model and two 2D fluoroscopic images. Comput. Vis. Image Und. 113:202-211, 2009.

${ }^{25}$ Lamecker, H., T. H. Wenckebach, and H.-C. Hege. Atlasbased 3D-shape reconstruction from X-ray images. In: Proceedings of the 2006 International Conference on Pattern Recognition 2006 (ICPR 2006). IEEE Computer Society, 2006, pp. 371-374.

${ }^{26}$ Langlotz, U., P. A. Grützner, K. Bernsmann, J. Kowal, M. Tannast, M. Caversaccio, and L.-P. Nolte. Accuracy considerations in navigated cup placement for THR. Proc. Inst. Mech. Eng. H 221:739-753, 2007.

${ }^{27}$ Laporte, S., W. Skalli, J. A. de Guise, F. Lavaste, and D. Mitton. A biplanar reconstruction method based on 2D and 3D contours: application to the distal femur. Comput. Methods Biomech. Biomed. Eng. 6:1-6, 2003.

${ }^{28}$ Larose, D., L. Cassenti, B. Jaramaz, J. E. Moody, T. Kanade, and A. M. DiGioia. Post-operative measurement of acetabular cup position using X-ray/CT registration. In: Proceedings of the $3 \mathrm{rd}$ International Conference on Medical Image Computing and Computer-assisted Intervention (MICCAI 2000), LNCS 1935:1104-1113, 2000.

${ }^{29}$ le Bras, A., S. Laporte, V. Bousson, D. Mitton, J. A. de Guise, J. D. Laredo, and W. Skalli. 3D reconstruction of the proximal femur with low-dose digital stereoradiography. Comput. Aided Surg. 9:51-57, 2004.

${ }^{30}$ Lewinnek, G. E., J. L. Lewis, R. Tarr, C. L. Compere, and J. R. Zimmerman. Dislocations after total hip-replacement arthroplasties. J. Bone Joint Surg. Am. 60(2):217-220, 1978.

${ }^{31}$ Lin, F., D. Lim, R. L. Wixson, S. Milos, R. W. Hendrix, and M. Makhsous. Validation of a computer navigation system and a CT method for determination of the orientation of implanted acetabular cup in total hip arthroplasty: a cadaver study. Clin. Biomech. 23:1004-1011, 2008.

${ }^{32}$ Mahfouz, M., A. Badawi, E. E. A. Fatah, M. Kuhn, and B. Merkl. Reconstruction of 3D patient-specific bone models from biplanar X-ray images utilizing morphometric measurements. In: Proceedings of the 2006 International Conference on Image Processing, Computer Vision, and Pattern Recognition (IPCV 2006), edited by H. R. Arabnia. Las Vegas, USA, 2006, pp. 345-349.

${ }^{33}$ Marx, A., M. von Knoch, J. Pförtner, M. Wiese, and G. Saxler. Misinterpretation of cup anteversion in total hip arthroplasty using planar radiography. Arch. Orthop. Trauma Surg. 126:487-492, 2006.

${ }^{34}$ McCollum, D. E., and W. J. G. Gray. Dislocation after total hip arthroplasty. Causes and prevention. Clin. Orthop. 261:159-170, 1990.

${ }^{35}$ Metz, C. E., and L. E. Fencil. Determination of threedimensional structure in biplane radiography without prior knowledge of the relationship between two views: theory. Med. Phys. 16(1):45-51, 1989.

${ }^{36}$ Mitton, D., S. Deschênes, S. Laporte, B. Godbout, S. Bertrand, J. A. de Guise, and W. Skalli. 3D reconstruction of the pelvis from bi-planar radiography. Comput. Methods Biomech. Bipmed. Eng. 9:1-5, 2006.

${ }^{37}$ Mitton, D., C. Landry, S. Véron, W. Skalli, F. Lavaste, and J. A. de Guise. 3D reconstruction method from biplanar radiography using non-stereocorresponding points and elastic deformable meshes. Med. Biol. Eng. Comput. 38:133-139, 2000.

${ }^{38}$ Mitton, D., K. Zhao, S. Bertrand, C. Zhao, S. Laporte, C. Yang, K.-N. An, and W. Skalli. 3D reconstruction of the ribs from lateral and frontal $\mathrm{X}$-rays in comparison to $3 \mathrm{D}$ CT-scan reconstruction. J. Biomech. 41(3):706-710, 2008.

${ }^{39}$ Murray, D. W. The definition and measurement of acetabular orientation. J. Bone Joint Surg. [Br.] 75-B:228232, 1993.

${ }^{40}$ Novosad, J., F. Cheriet, Y. Petit, and H. Labelle. Threedimensional (3-D) reconstruction of the spine from a single $\mathrm{X}$-ray image and prior vertebra models. IEEE Trans. Biomed. Eng. 51:1628-1638, 2004.

${ }^{41}$ Patil, S., A. Bergula, P. C. Chen, C. W. Colwell, Jr., and D. D. D'Lima. Polyethylene wear and acetabular component orientation. J. Bone Joint Surg. [ Am.] 85:56-63, 2003.

${ }^{42}$ Penney, G. P., P. J. Edwards, J. H. Hipwell, M. Slomczykowski, I. Revie, and D. J. Hawkes. Postoperative calculation of acetabular cup position using 2D-3D registration. IEEE Trans. Biomed. Eng. 54:1342-1348, 2007.

${ }^{43}$ Pomero, V., D. Mitton, S. Laporte, J. A. de Guise, and W. Skalli. Fast accurate stereoradiographic 3D-reconstruction of the spine using a combined geometric and statistic model. Clin. Biomech. 19:240-247, 2004.

${ }^{44}$ Pradhan, R. Planar anteversion of the acetabular cup as determined from plain anteroposterior radiographs. J. Bone Joint Surg. Br. 81:431-435, 1999.

${ }^{45}$ Sadowsky, O., G. Chintalapani, and R. Taylor. Deformable 2D-3D registration of the pelvis with a limited field of view, using shape statistics. In: Proceedings of the 10th International Conference on Medical Image Computing and Computer-assisted Interventions (MICCAI 2007), LNCS 4792:519-526, Springer 2007.

${ }^{46}$ Sarmiento, A., E. Ebramzadeh, W. J. Gogan, and H. A. McKellop. Cup containment and orientation in cemented total hip arthroplasties. J. Bone Joint Surg. Br. 72B(6):9961002,1990

${ }^{47}$ Sellers, R. G., D. Lyles, and L. D. Dorr. The effect of pelvic rotation on alpha and theta angles in total hip arthroplasty. Contemp. Orthop. 17:67-70, 1988.

${ }^{48}$ Small, C. G. The Statistical Theory of Shape. New York: Springer Series in Statistics, Springer-Verlag, 1996.

${ }^{49}$ Steppacher, S. D., M. Tannast, G. Zheng, X. Zhang, J. Kowal, S. Anderson, K. A. Siebenrock, and S. B. Murphy. Validation of a new method for determination of cup orientation in THA. J. Orthop. Res., 2009 (in press). doi:10.1002/jor.20929.

${ }^{50}$ Tannast, M., U. Langlotz, K. A. Siebenrock, K. M. Wiese, K. Bernsmann, and F. Langlotz. Anatomical referencing of 
cup orientation in total hip arthroplasty. Clin. Orthop. Relat. Res. 436:144-150, 2005.

${ }^{51}$ Tannast, M., G. Zheng, C. Anderegg, K. Burckhardt, F. Langlotz, R. Ganz, and K. A. Siebenrock. Tilt and rotation correction of acetabular version on pelvic radiographs. Clin. Orthop. Relat. Res. 438:182-190, 2005.

${ }^{52}$ The, B., R. L. Diercks, R. E. Stewart, P. M. A. van Ooijen, and J. R. van Horn. Digital correction of magnification in pelvic x-rays for preoperative planning of hip joint replacement: theoretical development and clinical results of a new protocol. Med. Phys. 32(8):2580-2589, 2005.

${ }^{53}$ Thirion, J.-P. Image matching as a diffusion process: an analogy with Maxwell's demons. Med. Image Anal. 2:243260, 1998.

${ }^{54}$ Toussaint, N., J.-C. Souplet, and P. Fillard. MedINRIA: medical image navigation and research tool by INRIA. In: Proceedings of MICCAI'07 Workshop on Interaction in Medical Image Analysis and Visualization, Brisbane, Australia, 2007.

${ }^{55}$ Turk, M., and A. Pentland. Eigenfaces for recognition. J. Cogn. Neurosci. 3:71-86, 1991.

${ }^{56}$ Veldpaus, F. E., H. J. Woltring, and L. J. M. G. Dortmans. A least-square algorithm for the equiform transformation from spatial marker co-ordinates. J. Biomech 21:45-54, 1988.

${ }^{57}$ Widmer, K.-H. A simplified method to determine acetabular cup anteversion from plain radiographs. J. Arthroplasty 19:387-390, 2004.

${ }^{58}$ Widmer, K. H. Containment versus impingement: finding a compromise for cup placement in total hip arthroplasty. Int. Orthop. 31(Suppl 1):S29-S33, 2007.

${ }^{59}$ Zheng, G. Statistically deformable $2 \mathrm{D} / 3 \mathrm{D}$ registration for accurate determination of post-operative cup orientation from single standard X-ray radiograph. In: Proceedings of the 12th International Conference on Medical Image Computing and Computer-assisted Interventions (MICCAI 2009), LNCS 5761:820-827, Springer 2009.

${ }^{60}$ Zheng, G., X. Dong, and M. A. Gonzalez Ballester. Unsupervised reconstruction of a patient-specific model of a proximal femur from calibrated fluoroscopic images. In: Proceedings of the 10th International Conference on Medical Image Computing and Computer-assisted Interventions (MICCAI 2007), LNCS 4791:834-841, Springer 2007.

${ }^{61}$ Zheng, G., X. Dong, K. Rajamani, X. Zhang, M. Styner, R. Thoranaghatte, L.-P. Nolte, and M. A. González Ballester. Accurate and robust reconstruction of a surface model of the proximal femur from sparsepoint data and dense-point distribution model for surgical navigation. IEEE Trans. Biomed. Eng. 54:2109-2122, 2007.

${ }^{62}$ Zheng, G., S. Gollmer, S. Schumann, X. Dong, T. Feilkas, and M. A. Gonzalez Ballester. A 2D/3D correspondence building method for reconstruction of a patient-specific $3 \mathrm{D}$ bone surface model using point distribution models and calibrated X-ray images. Med. Image Anal. 13(6):883-899, 2009.

${ }^{63}$ Zheng, G., M. A. Gonzalez Ballester, M. Styner, and L.-P. Nolte. Reconstruction of patient-specific 3D bone surface from 2D calibrated fluoroscopic images and point distribution model. In: Proceedings of the 9th International Conference on Medical Image Computing and Computerassisted Interventions (MICCAI 2006), LNCS 4190:25-32, Springer 2006.

${ }^{64}$ Zheng, G., S. Steppacher, X. Zhang, and M. Tannast. Precise estimation of postoperative cup alignment from single standard X-ray radiograph with gonadal shielding. In: Proceedings of 2007 International Conference on Medical Image Computing and Computer Assisted Intervention (MICCAI 2007), Part II, LNCS 4792:951-959, Springer 2007.

${ }^{65}$ Zheng, G., X. Zhang, S. Steppacher, S. B. Murphy, K. A. Siebenrock, and M. Tannast. HipMatch: an object-oriented cross-platform program for accurate determination of cup orientation using 2D-3D registration of single standard $\mathrm{X}$-ray radiograph and a $\mathrm{CT}$ volume. Comput. Methods Programs Biomed. 95:236-248, 2009. 